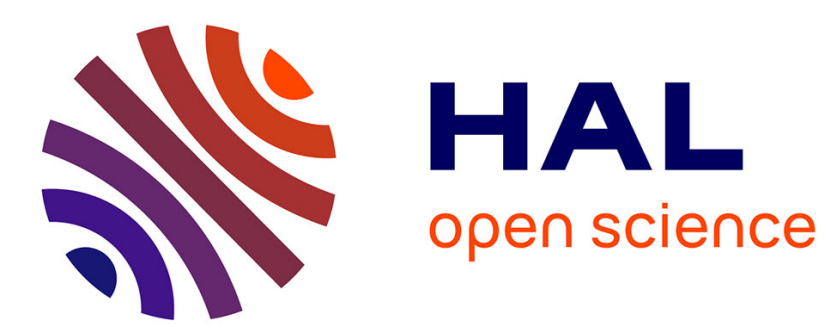

\title{
GENERALIZED PUNCTUAL HILBERT SCHEMES AND g-COMPLEX STRUCTURES
}

\author{
Alexander Thomas
}

\section{To cite this version:}

Alexander Thomas. GENERALIZED PUNCTUAL HILBERT SCHEMES AND g-COMPLEX STRUCTURES. 2020. hal-02455735

\author{
HAL Id: hal-02455735 \\ https://hal.science/hal-02455735
}

Preprint submitted on 26 Jan 2020

HAL is a multi-disciplinary open access archive for the deposit and dissemination of scientific research documents, whether they are published or not. The documents may come from teaching and research institutions in France or abroad, or from public or private research centers.
L'archive ouverte pluridisciplinaire HAL, est destinée au dépôt et à la diffusion de documents scientifiques de niveau recherche, publiés ou non, émanant des établissements d'enseignement et de recherche français ou étrangers, des laboratoires publics ou privés. 


\title{
GENERALIZED PUNCTUAL HILBERT SCHEMES AND $\mathfrak{g}$-COMPLEX STRUCTURES
}

\author{
ALEXANDER THOMAS
}

\begin{abstract}
In this paper we define and analyze a generalization of the punctual Hilbert scheme of the plane which is associated to a simple complex Lie algebra $\mathfrak{g}$. Using this generalized punctual Hilbert scheme, we construct a new geometric structure on a surface, called a $\mathfrak{g}$-complex structure, generalizing complex structures and higher complex structures from [FT19]. We investigate the geometry of the $\mathfrak{g}$-complex structure and define a moduli space which conjecturally is isomorphic to Hitchin's component of the character variety for the real split group G.
\end{abstract}

\section{INTRODUCTION}

The main motivation for this paper is to get a geometric approach to Hitchin components. These components were constructed by Nigel Hitchin in his famous paper [Hi92] using analytic methods (Higgs bundles). Hitchin components are connected components of the character variety $\operatorname{Hom}\left(\pi_{1}(\Sigma), G\right) / G$ where $\Sigma$ is a smooth surface, closed and without boundary and $G$ is a adjoint group of a split real form of a complex simple Lie group.

For the group $G=P S L_{2}(\mathbb{R})$, we obtain Teichmüller space which is also the moduli space of complex structures on $\Sigma$. For $G=P S L_{n}(\mathbb{R})$, Vladimir Fock and the author defined in [FT19] a new geometric structure, called higher complex structure, whose moduli space is conjecturally isomorphic to Hitchin's component. The main ingredient to construct the higher complex structure is the punctual Hilbert scheme of the plane and its zero-fiber.

In this article, we pursue these ideas by defining a $\mathfrak{g}$-complex structure, for a complex simple Lie algebra $\mathfrak{g}$, using a generalization of the punctual Hilbert scheme, which we call $\mathfrak{g}$-Hilbert scheme.

Our strategy to define these new objects is twofold: on the one hand we use the various descriptions of the punctual Hilbert scheme, especially the matrix viewpoint, in order to generalize to an arbitrary $\mathfrak{g}$. On the other, we got inspiration from Hitchin's original paper Hi92] (section 5) where he starts with a principal nilpotent element and deforms it into an element of a principal slice, a generalized companion matrix.

We signal to the reader that our definition of the $\mathfrak{g}$-Hilbert scheme might get changed in the future, since as it is defined now, it is a non-Hausdorff space. There should be a way to get a nice topological space, without loosing any of its properties. This possible modification will not affect the $\mathfrak{g}$-complex structure since only the regular part of the $\mathfrak{g}$-Hilbert scheme plays a role in its construction.

Université de Strasbourg, IRMa UMR 7501, 67084 Strasbourg, France

E-mail address: thomas@math.unistra.fr. 
The outline of the paper is the following: In the first section 1, we define the generalized punctual Hilbert scheme, and some interesting subsets, for example the zero-fiber, the regular and the cyclic part. We analyze the relations between these parts and define a generalized Chow map. For classical $\mathfrak{g}$ we also define a map to a space of ideals and we analyze in detail the regular part of the $\mathfrak{g}$-Hilbert scheme. Finally, we study the topology of the $\mathfrak{g}$-Hilbert scheme and formulate some conjectures. In section 2, we construct the $\mathfrak{g}$-complex structure and show how it induces in a natural way a complex structure. We give an equivalent construction using ideals for classical $\mathfrak{g}$. In section 3 , for $\mathfrak{g}$ of classical type, we define the notion of higher diffeomorphism of type $\mathfrak{g}$, which gives a definition of a moduli space of $\mathfrak{g}$-complex structures. We then explore its properties which show its similarity to Hitchin's component. In particular we define a spectral curve in the complexified cotangent bundle $T^{* \mathbb{C}} \Sigma$. In the final part 4 we give a larger conjectural picture which would give the link to Hitchin components. We include two appendices: In appendix A, we review the main properties of the punctual Hilbert scheme of the plane. In the second appendix $B$, we gather all properties we need in the paper of regular elements in semisimple Lie algebras and give precise references.

Notations. Throughout the paper, $\Sigma$ denotes a smooth surface, closed, without boundary and orientable. We denote by $\mathfrak{g}$ a complex simple Lie algebra, by $\mathfrak{h}$ a Cartan subalgebra, by $W$ its Weyl group and by $G$ its adjoint group (the unique Lie group $G$ with Lie algebra $\mathfrak{g}$ with trivial center).

Acknowledgments. The author wish to express his gratitude towards Vladimir Fock, for all the fruitful discussions. I also thank Loren Spice for answering a question of mine concerning connectedness of stabilizers on mathoverflow.

\section{Generalized punctual Hilbert scheme}

In this section, we generalize the punctual Hilbert scheme to a $\mathfrak{g}$-Hilbert scheme and explore the properties of the new object. We analyze in detail its regular part in the case of a classical Lie algebra. The reader not familiar with punctual Hilbert schemes should consult Appendix A.

1.1. Definitions and first properties. The punctual Hilbert scheme $\operatorname{Hilb}^{n}\left(\mathbb{C}^{2}\right)$ has several descriptions:

- as a space of ideals (the idealic viewpoint)

- as a desingularization of the configuration space $\mathfrak{h}^{2} / W$ for $\mathfrak{g}=\mathfrak{g l}_{n}$

- as a space of commuting matrices (the matrix viewpoint).

It is the matrix viewpoint which will be generalized. So let us recall it quickly here:

$\operatorname{Hilb}^{n}\left(\mathbb{C}^{2}\right) \cong\left\{(A, B) \in \mathfrak{g l}_{n}^{2} \mid[A, B]=0,(A, B)\right.$ admits a cyclic vector $\} / G L_{n}$.

The main difficulty is to find an intrinsic condition which generalizes the existence of a cyclic vector. Here is our proposal:

Definition 1.1. The generalized punctual Hilbert scheme, or $\mathfrak{g}$-Hilbert scheme, denoted by $\operatorname{Hilb}(\mathfrak{g})$, is defined by

$$
\operatorname{Hilb}(\mathfrak{g})=\left\{(A, B) \in \mathfrak{g}^{2} \mid[A, B]=0, \operatorname{dim} Z(A, B)=\operatorname{rk} \mathfrak{g}\right\} / G
$$

where $Z(A, B)$ denotes the common centralizer of $A$ and $B$, i.e. the set of elements $C \in \mathfrak{g}$ which commute with $A$ and $B$. 
The condition on the dimension of the common centralizer does not come from nowhere: Proposition B.9 of Appendix B shows that $\mathrm{rkg}$ is the minimal possible dimension for the centralizer of a commuting pair. Define the commuting variety by $\operatorname{Comm}(\mathfrak{g})=\left\{(A, B) \in \mathfrak{g}^{2} \mid[A, B]=0\right\}$. The $\mathfrak{g}$-Hilbert scheme is the set of all regular points of $\operatorname{Comm}(\mathfrak{g})$ modulo $G$.

Remark. Ginzburg has defined the notion of a principal nilpotent pair in [Gi99], which is more restrictive than ours. He calls "nil-pairs" elements of our $\mathfrak{g}$-Hilbert scheme, but he does not investigate them.

Let us give two examples of elements in the $\mathfrak{g}$-Hilbert scheme:

Example 1.2. Let $A \in \mathfrak{g}$ be a regular element. Then by a theorem of Kostant (see B.6), its centralizer $Z(A)$ is abelian. So for any $B \in Z(A)$, we have $Z(A) \subset Z(B)$, thus $Z(A, B)=Z(A) \cap Z(B)=Z(A)$ is of dimension rk $\mathfrak{g}$. Therefore $[(A, B)] \epsilon$ $\operatorname{Hilb}(\mathfrak{g})$.

If $A$ is principal nilpotent, then $B \in Z(A)$ is also nilpotent. So $[(A, B)] \in \operatorname{Hilb}_{0}(\mathfrak{g})$, the zero-fiber defined below.

If $B=0$ then $[(A, 0)]$ is in $\operatorname{Hilb}(\mathfrak{g})$ iff $A$ is regular.

Example 1.3. Let $(A, B)$ be a commuting pair of matrices in $\mathfrak{s l}_{n}$ admitting a cyclic vector, i.e. an element of the reduced Hilbert scheme. One way to get such a pair is the following construction: take a Young diagram (our convention is to put the origin in the upper left corner as for matrices) with $n$ boxes (see figure 1). Associate to each box a vector of a basis of $\mathbb{C}^{n}$. Define $A$ to be the matrix which translates to the left, i.e. sends a vector to the vector in the box to the left or to 0 if there is none. Let $B$ be the matrix which translates to the bottom. Then $A$ and $B$ clearly commute and are nilpotent. In proposition 1.5 below, we show that $Z(A, B)$ is of minimal dimension in that case.
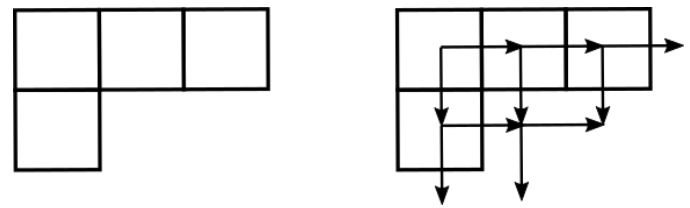

FIGURE 1. Young diagram and commuting nilpotent matrices

Guided by these examples, we define several subsets of the $\mathfrak{g}$-Hilbert scheme and explore their relations. First, we define the zero-fiber and the regular part which will both play a mayor role in the definition of a $\mathfrak{g}$-complex structure. We also define the cyclic part, which is not intrinsically defined since it uses a representation of $\mathfrak{g}$. The cyclic part will be used to define a map to a space of ideals, getting a generalization of the original description of the punctual Hilbert scheme.

Definition 1.4. The zero-fiber of the $\mathfrak{g}$-Hilbert scheme is defined by

$$
\operatorname{Hilb}_{0}(\mathfrak{g})=\{[(A, B)] \in \operatorname{Hilb}(\mathfrak{g}) \mid A \text { and } B \text { nilpotent }\} \text {. }
$$

We define the regular part of the $\mathfrak{g}$-Hilbert scheme, denoted by $\operatorname{Hilb}^{\text {reg }}(\mathfrak{g})$, to be those conjugacy classes $[(A, B)]$ in which $A$ or $B$ is a regular element of $\mathfrak{g}$.

Finally for classical $\mathfrak{g}$, let $\rho$ denote the natural representation of $\mathfrak{g}$ (i.e. $\mathfrak{s l}_{n} \subset$ $\mathfrak{g l}_{n}, \mathfrak{s o}_{n} \subset \mathfrak{g l}_{n}$ and $\left.\mathfrak{s p}_{2 n} \subset \mathfrak{g l}_{2 n}\right)$. Define the cyclic part of the $\mathfrak{g}$-Hilbert scheme by

$\operatorname{Hilb}^{\text {cycl }}(\mathfrak{g})=\left\{(A, B) \in \mathfrak{g}^{2} \mid[A, B]=0,(\rho(A), \rho(B))\right.$ admits a cyclic vector $\} / G$. 
Remark. In the definition of the cyclic part, it would be more natural to consider the adjoint representation, but even in the case of $\mathfrak{s l}_{2}$, this would give a map to a space of ideals, which is not the one of $\mathrm{Hilb}_{\text {red }}^{2}\left(\mathbb{C}^{2}\right)$.

Instead of the standard representation, one could also use a non-trivial representation of minimal dimension, which for classical $\mathfrak{g}$ is always the standard representation, apart from type $D_{3}$ and $D_{4}$. For $D_{3}$, the two spin representations are of minimal dimension, and they give an isomorphism between $\mathfrak{s o}_{6}$ and $\mathfrak{s l}_{4}$. For type $D_{4}$, there are three representations of minimal dimension, the standard one and the two spin representations. All of them are linked by outer automorphisms coming from the symmetry of the Dynkin diagram. Thus the cyclic part of the $\mathfrak{g}$-Hilbert scheme is the same for all three representations.

Taking the representation of minimal dimension has the additional advantage to be well-defined for all $\mathfrak{g}$. We have not computed any example of an exceptional Lie algebra, so we do not know whether the use of the representation of minimal dimension is of interest.

The first relation between the various Hilbert schemes is the inclusion of the cyclic part in the $\mathfrak{g}$-Hilbert scheme, which justifies the name "cyclic part":

Proposition 1.5. For $\mathfrak{g}$ of classical type, we have $\operatorname{Hilb}^{\text {cycl }}(\mathfrak{g}) \subset \operatorname{Hilb}(\mathfrak{g})$.

Proof. Recall $\rho$ the natural representation of $\mathfrak{g}$ on $\mathbb{C}^{m}$. For simplicity, we write $A$ instead of $\rho(A)$ here.

Let $(A, B) \in \mathfrak{g}^{2}$ admitting a cyclic vector $v$. Let $C \in Z(A, B)$. Then $C$ is a polynomial in $A$ and $B$. Indeed, there is $P \in \mathbb{C}[x, y]$ such that $C v=P(A, B) v$. Since $C$ commutes with $A$ and $B$, we then get for any polynomial $Q$ that $C Q(A, B) v=$ $Q(A, B) C v=Q(A, B) P(A, B) v=P(A, B) Q(A, B) v$, so $C=P(A, B)$.

Therefore the common centralizer of $(A, B)$ in $\mathfrak{g l}_{m}$ is $\mathbb{C}[A, B] / I$ where $I=\{P \in$ $\mathbb{C}[x, y] \mid P(A, B)=0\}$. We know from Appendix A that $I$ is of codimension $m$ since $(A, B)$ admits a cyclic vector. We have $Z(A, B)=Z_{\mathfrak{g l}_{m}}(A, B) \cap \mathfrak{g}$. One can easily check that for $\mathfrak{g}$ of type $A_{n}$, a polynomial $P(A, B)$ is in $\mathfrak{g}$ iff its constant term has a specific form, given by the other coefficients (to ensure trace zero). For type $B_{n}, C_{n}$ and $D_{n}, P(A, B)$ is in $\mathfrak{g}$ iff $P$ is odd. One checks in each case that the dimension of $Z(A, B)$ equals the rank of $\mathfrak{g}$.

In general, the inclusion of the cyclic Hilbert scheme is strict as shows the following example:

Example 1.6. Consider $A=\left(\begin{array}{lll}0 & 1 & 0 \\ 0 & 0 & 0 \\ 0 & 0 & 0\end{array}\right)$ and $B=\left(\begin{array}{lll}0 & 0 & 1 \\ 0 & 0 & 0 \\ 0 & 0 & 0\end{array}\right)$ in $\mathfrak{s l}_{3}$. One easily checks that the pair $(A, B)$ does not admit any cyclic vector, but that their common centralizer is of dimension 2. So $[(A, B)] \in \operatorname{Hilb}\left(\mathfrak{s l}_{3}\right) \backslash \operatorname{Hilb}^{\text {cycl }}\left(\mathfrak{s l}_{3}\right)$.

This example will be used in subsection 1.9 to show that Hilb $(\mathfrak{g})$ is not Hausdorff.

In general, there is no link between regular and cyclic part. Example 1.3 shows that cyclic elements are not always regular and the following example shows that regular element are not always cyclic:

Example 1.7. For $\mathfrak{g}$ of type $D_{n}$, let $f$ be a principal nilpotent element. Then one checks that $[(f, 0)] \in \operatorname{Hilb}\left(\mathfrak{s o}_{2 n}\right)$ is regular but not cyclic (see also subsection 1.8 ).

Let us turn to the regular part. It turns out that if one fixes a principal slice $f+Z(e)$ in $\mathfrak{g}$ (see Appendix $\mathrm{B}$ ), there is a preferred representative for regular classes: 
Proposition 1.8. Any class $[(A, B)] \in \operatorname{Hilb}^{\text {reg }}(\mathfrak{g})$ where $A$ is regular can uniquely be conjugated to $(A \in f+Z(e), B \in Z(A))$.

Proof. By the property of the principal slice, there is a unique conjugate of $A$ which is in the principal slice $f+Z(e)$. Denote still by $A$ and $B$ these conjugates. The only thing to show is that $B$ is unique which is done in the next lemma.

Lemma 1.9. If $A \in \mathfrak{g}$ is regular, $g \in G$ such that $A d_{g}(A)=A$ and $B \in Z(A)$, then $A d_{g}(B)=B$.

Proof. By Kostant's theorem B.6, we know that $Z(A)$ is abelian. So the infinitesimal version of the lemma is true. We conclude by the connectedness of the stabilizer of $A$, given by the next lemma.

Lemma 1.10. For a regular element $A \in \mathfrak{g}$, its stabilizer $\operatorname{Stab}(A)=\{g \in G \mid$ $\left.A d_{g}(A)=A\right\}$ in the adjoint group $G$ is connected.

Proof. Decompose $A$ into Jordan form: $A=A_{s}+A_{n}$ with $A_{s}$ semisimple, $A_{n}$ nilpotent and $\left[A_{s}, A_{n}\right]=0$. So $A_{n} \in Z\left(A_{s}\right)$. The structure of the centralizer $Z\left(A_{s}\right)$ is wellknown: it is a direct sum of a Cartan $\mathfrak{h}$ containing $A_{s}$ with all root spaces $\mathfrak{g}_{\alpha}$ where $\alpha$ is a root such that $\alpha\left(A_{s}\right)=0$. It is also known that $Z\left(A_{s}\right)$ is reductive, so a direct sum $Z\left(A_{s}\right)=\mathfrak{c} \oplus \mathfrak{g}_{s}$ where $\mathfrak{c}$ is the center and $\mathfrak{g}_{s}$ is the semisimple part of $Z\left(A_{s}\right)$. In particular the center $\mathfrak{c}$ is included in $\mathfrak{h}$. So $A_{n} \in \mathfrak{g}_{s}$ since $A_{n}$ is nilpotent. Denote by $G_{s}$ the Lie group with trivial center with Lie algebra $\mathfrak{g}_{s}$.

We know that $A$ is regular is equivalent to $A_{n}$ being regular nilpotent in $\mathfrak{g}_{s}$ (see Ko63, proposition 0.4). We also know that the $G$-equivariant fundamental group of the orbit of $A$ (which is the space of connected components of $\operatorname{Stab}(A)$ ) is the same as the $\operatorname{Stab}\left(A_{s}\right)$-equivariant fundamental group of the $\operatorname{Stab}\left(A_{s}\right)$-orbit of $A_{n}$ (see Proposition 6.1.8. of [CM93] adapted to the adjoint group). In other words, the connected components of $\operatorname{Stab}_{G}(A)$ are the same as the connected components of $\operatorname{Stab}_{G_{s}}\left(A_{n}\right)$ since the $\operatorname{Stab}\left(A_{s}\right)$-orbit of $A_{n}$ is equal to the $G_{s}$-orbit of $A_{n}$.

So we are reduced to the principal nilpotent case. Using the classification of simple Lie algebras, one can check explicitly in Collingwood-McGovern's book [CM93] the tables 6.1.6. for classical $\mathfrak{g}$ and the tables at the end of chapter 8 for exceptional $\mathfrak{g}$ that the stabilizer of a principal nilpotent element is always connected.

Remark. It is surprising that the last lemma has never been stated (at least not to our knowledge). It would be interesting to find a direct argument, without using the classification of simple Lie algebras.

Corollary 1.11. The regular zero-fiber $\operatorname{Hilb}_{0}^{\text {reg }}(\mathfrak{g})=\operatorname{Hilb}^{\text {reg }}(\mathfrak{g}) \cap \operatorname{Hilb}_{0}(\mathfrak{g})$ is an affine variety of dimension rk $\mathfrak{g}$.

Proof. This follows directly from the previous proposition using the fact that $A \epsilon$ $f+Z(e)$ is nilpotent iff $A=f$. So Hilb ${ }_{0}^{r e g}(\mathfrak{g})$ is described by $Z(f)$ which is a vector space of dimension rk $\mathfrak{g}$.

We know that both the regular and the cyclic part are in general strictly included in the $\mathfrak{g}$-Hilbert scheme. But they are dense subspaces:

Proposition 1.12. The regular part $\operatorname{Hilb}^{\text {reg }}(\mathfrak{g})$ is dense in $\operatorname{Hilb}(\mathfrak{g})$. For classical $\mathfrak{g}$, the cyclic part is also dense in $\operatorname{Hilb}(\mathfrak{g})$. 
Proof. By a theorem of Richardson (see B.8), the set of semisimple commuting pairs is dense in the commuting variety $\operatorname{Comm}(\mathfrak{g})$. So the set of semisimple regular elements is also dense in $\operatorname{Comm}(\mathfrak{g})$. Passing to the quotient by $G$, we get that the classes of semisimple regular pairs are dense in $\operatorname{Hilb}(\mathfrak{g})$ since $\operatorname{Hilb}(\mathfrak{g}) \subset \operatorname{Comm}(\mathfrak{g}) / G$ and all semisimple regular pairs are in $\operatorname{Hilb}(\mathfrak{g})$. Since the semisimple regular pairs are in the regular part, we get the density of $\operatorname{Hilb}^{r e g}(\mathfrak{g})$ in $\operatorname{Hilb}(\mathfrak{g})$.

For classical $\mathfrak{g}$, we have the same argument for the cyclic part since semisimple regular pairs are cyclic.

To end the section, we state an analogue of Kostant's theorem about abelian subalgebras of centralizers:

Proposition 1.13. For any commuting pair $(A, B) \in \operatorname{Comm}(\mathfrak{g})$, there is an abelian subspace of dimension rk $\mathfrak{g}$ in the common centralizer $Z(A, B)$.

Proof. The proof is completely analogous to Kostant's proof for theorem B.6: we use a limit argument. Let $\left(A_{n}, B_{n}\right)$ be a sequence of regular semisimple pairs converging to $(A, B)$ (exists since regular semisimple pairs are dense). We know that $Z\left(A_{n}, B_{n}\right)$ is a rk $\mathfrak{g}$-dimensional abelian subspace of $\mathfrak{g}$. Since the Grassmannian $\operatorname{Gr}(\mathrm{rk} \mathfrak{g}, \operatorname{dim} \mathfrak{g})$ is compact, there is a subsequence of $Z\left(A_{n}, B_{n}\right)$ which converges. It is easy to prove that the limit is included in $Z(A, B)$ and is commutative.

Corollary 1.14. For $[(A, B)] \in \operatorname{Hilb}(\mathfrak{g})$, the common centralizer $Z(A, B)$ is abelian.

Remark. For classical $\mathfrak{g}$, the corollary is easy for the cyclic part since $Z(A, B)=$ $\mathbb{C}[x, y] / I \cap \mathfrak{g}$ which is abelian since $\mathbb{C}[x, y]$ is.

In the following sections, we generalize as far as possible the other viewpoints of the usual Hilbert scheme (resolution of configuration space and idealic viewpoint) to our setting.

1.2. Chow map. We want to generalize the Chow map, which goes from $\operatorname{Hilb}^{n}\left(\mathbb{C}^{2}\right)$ to the configuration space (see A.2).

Fix a Cartan subalgebra $\mathfrak{h}$ in $\mathfrak{g}$. Recall the Jordan decomposition in a semisimple Lie algebra: for $x \in \mathfrak{g}$, there is a unique pair $\left(x_{s}, x_{n}\right)$ with $x=x_{s}+x_{n}, x_{s}$ semisimple, $x_{n}$ nilpotent and $\left[x_{s}, x_{n}\right]=0$. For a semisimple element $x$, denote by $x^{*}$ a conjugate in the Cartan $\mathfrak{h}$ (unique up to $W$-action).

The Chow map $c h: \operatorname{Hilb}(\mathfrak{g}) \rightarrow \mathfrak{h}^{2} / W$ is defined by

$$
\operatorname{ch}([(A, B)])=\left[\left(A_{s}^{*}, B_{s}^{*}\right)\right]
$$

where the brackets [.] always denotes the equivalence class. For semisimple regular pairs, this map corresponds to a simultaneous diagonalization.

Proposition 1.15. The Chow map ch is well-defined and continuous.

Proof. Since $[A, B]=0$, we also have $\left[A_{s}, B_{s}\right]=0$ by a simultaneous Jordan decomposition in a faithful representation. Hence there is a conjugate of the pair $\left(A_{s}, B_{s}\right)$ which lies in $\mathfrak{h}^{2}$. Since the adjoint action of $G$ on $\mathfrak{g}$ restricts to the $W$-action on $\mathfrak{h}$, the map $c h$ is well-defined.

The map $x \mapsto x_{s}^{*}$ is continuous which simply follows from the continuity of eigenvalues. Hence the Chow map is continuous as well. 
Remark. The Jordan decomposition $x \mapsto\left(x_{s}, x_{n}\right)$ is not continuous at all, since semisimple elements are dense in $\mathfrak{g}$ for which we have $x_{n}=0$ andt for all nonsemisimple elements we have $x_{n} \neq 0$. But the map $x \mapsto x_{s}$ is continuous.

This map permits to think of a generic element of $\operatorname{Hilb}(\mathfrak{g})$ as a point in $\mathfrak{h}^{2} / W$, or via a representation of $\mathfrak{g}$ on $\mathbb{C}^{m}$, as a set of $m$ points in $\mathbb{C}^{2}$ with a certain symmetry. For $\mathfrak{g}=\mathfrak{s l}_{n}$ for example, these are $n$ points with barycenter 0 .

Since Hilb $(\mathfrak{g})$ is even not Hausdorff (see subsection 1.9), it cannot be a nonsingular variety. Nevertheless we conjecture the following:

Conjecture 1.16. There is a modified version of $\operatorname{Hilb}(\mathfrak{g})$, identifying some points, which is a smooth projective variety such that the Chow morphism is a resolution of singularities.

1.3. Idealic map. For $\mathfrak{g}$ of classical type, we can associate to any regular element of the $\mathfrak{g}$-Hilbert scheme an ideal, which we call idealic map. In this subsection, $\mathfrak{g}$ is a classical Lie algebra. Recall the natural representation $\rho$ of $\mathfrak{g}$ on $\mathbb{C}^{m}$ (see definition 1.4). We will write $A$ instead of $\rho(A)$.

We wish to define a map like in A.2.

$$
[(A, B)] \mapsto I(A, B)=\{P \in \mathbb{C}[x, y] \mid P(A, B)=0\} .
$$

If $[(A, B)] \in \operatorname{Hilb}^{c y c l}(\mathfrak{g})$ is cyclic, this ideal is of codimension $m$. But if the pair is not cyclic, there is no reason why the codimension should be $m$. In fact, there are examples for $\mathfrak{g}$ of type $D_{n}$ where the codimension is smaller.

We wish the idealic map to be continuous, so $I$ has to be of constant codimension. A strategy would be to define the idealic map $I$ on the cyclic part Hilb ${ }^{\text {cycl }}(\mathfrak{g})$ (which is dense by proposition 1.12 and to extend it by continuity. Unfortunately, the map can not be extended in a continuous way as shown in the following example:

Example 1.17. Take $\mathfrak{g}$ of type $D_{n}$. Denote by $f$ a principal nilpotent element. The pair $[(f, 0)] \in \operatorname{Hilb}\left(\mathfrak{s o}_{2 n}\right)$ is not cyclic (seen in example 1.7). Using the matrix $S$ defined in equation (1.4), we can approach $(f, 0)$ by $(f, t S)$ or by $\left(f+t S^{T}, 0\right)$ for $t \in \mathbb{C}^{\times}$going to 0 . These pairs are all cyclic. In the first case, the ideal is $I=\left\langle x^{2 n-1}, x y, y^{2}=t^{2} x^{2 n-2}\right\rangle$ which converges as $t$ goes to 0 to $\left\langle x^{2 n-1}, x y, y^{2}\right\rangle$. In the second case, the ideal is $I=\left\langle x^{2 n}+t^{2}, y\right\rangle$ converging to $\left\langle x^{2 n}, y\right\rangle$.

Because of this difficulty, our strategy is to define a space of ideals $I_{\mathfrak{g}}\left(\mathbb{C}^{2}\right)$, then a map Hilb ${ }^{\text {cycl }}(\mathfrak{g}) \rightarrow I_{\mathfrak{g}}\left(\mathbb{C}^{2}\right)$ and to extent it over the regular part Hilb ${ }^{\text {reg }}(\mathfrak{g})$ (in a non-continuous way). The last step is only necessary for $\mathfrak{g}$ of type $D_{n}$ since for the other classical types the regular part is included in the cyclic part as we will see in the sequel. The extension for $D_{n}$ will be defined ad hoc in subsection 1.8.

The previous section taught us to think of a generic element of $\operatorname{Hilb}(\mathfrak{g})$ as a $m$ tuple of points in $\mathbb{C}^{2}$ invariant under the Weyl group $W$. For type $A_{n}$ this means that the barycenter of the points is the origin. For the other classical types, this means that the set of points is symmetric with respect to the origin. Thus the defining ideal of these points is also invariant under the action of $W$. Hence the following definition.

Definition 1.18. We define the space of ideals of type $\mathfrak{g}$, denoted by $I_{\mathfrak{g}}\left(\mathbb{C}^{2}\right)$, to be the set of ideals in $\mathbb{C}[x, y]$ which are of codimension $m$ and $W$-invariant. For type $B_{n}, C_{n}$ and $D_{n}$ this means that $I$ is invariant under $(x, y) \mapsto(-x,-y)$. 
The map $I: \operatorname{Hilb}^{\text {cycl }}(\mathfrak{g}) \rightarrow I_{\mathfrak{g}}\left(\mathbb{C}^{2}\right)$ given by equation $(1.1)$ above is well-defined. Indeed, the codimension is $m$ by cyclicity and the ideal is $W$-invariant since this is a closed condition and it is true on the dense subset of regular semisimple pairs.

Notice that $I_{\mathfrak{g}}\left(\mathbb{C}^{2}\right)$ is the same for $\mathfrak{g}$ of type $C_{n}$ or $D_{n}$. But we will see that the idealic map $I$ has not the same image in the two cases. We will also see that for $\mathfrak{g}$ of type $A_{n}, B_{n}$ or $C_{n}$ the idealic map is injective. But for type $D_{n}$ it is not (it is generically 2 to 1 ). This comes from the fact that the Weyl group acting on the generic $2 n$ points, coming in $n$ pairs $\left(P_{i}, P_{i+1}=-P_{i}\right)$, cannot exchange $P_{1}$ and $P_{2}$ while leaving all other points fixed.

As for the usual Hilbert scheme, there is a direct link between the idealic map and the Chow morphism:

Proposition 1.19. The Chow map ch is the composition of the idealic map with the map which associates to an ideal its support, seen as an element of $\mathfrak{h}^{2} / W$ :

$$
\operatorname{ch}([(A, B)])=\operatorname{supp} I(A, B) .
$$

Proof. The statement is true on regular semisimple pairs which is a dense subset. For $\mathfrak{g}$ of type $A_{n}, B_{n}$ and $C_{n}$, it follows by continuity of both the Chow map and the idealic map. For $D_{n}$, our definition of the idealic map is to pick one of the various possible limits. In particular, the support of the ideal is still given by the Chow map.

1.4. Morphisms. In this subsection, we analyze the functorial behavior of the $\mathfrak{g}$ Hilbert scheme. In particular we construct two maps linked to the zero-fiber of the Hilbert scheme of $\mathfrak{s l}_{2}$ which will lead in the construction of the moduli space $\hat{\mathcal{T}}_{\mathfrak{g}} \Sigma$ of $\mathfrak{g}$-complex structures to maps from and to Teichmüller space.

Let $\psi: \mathfrak{g}_{1} \rightarrow \mathfrak{g}_{2}$ be a morphism of Lie algebras. For $[(A, B)] \in \operatorname{Hilb}\left(\mathfrak{g}_{1}\right)$, we can associate $[(\psi(A), \psi(B))]$ which is a well-defined map to $\operatorname{Comm}\left(\mathfrak{g}_{2}\right) / G_{2}$. But there is no reason why $\operatorname{dim} Z(\psi(A), \psi(B))$ should be minimal.

If we accept conjecture 1.16, that there is a modified version of the $\mathfrak{g}$-Hilbert scheme which is a resolution of $\mathfrak{h}^{2} / W$, we have a functorial behavior:

Proposition 1.20. Assuming conjecture 1.16, there is an induced map $\operatorname{Hilb}\left(\mathfrak{g}_{1}\right) \rightarrow$ $\operatorname{Hilb}\left(\mathfrak{g}_{2}\right)$.

Proof. Choose Cartan subalgebras $\mathfrak{h}_{1}$ and $\mathfrak{h}_{2}$ such that $\psi\left(\mathfrak{h}_{1}\right)=\mathfrak{h}_{2}$. Consider the composition $\mathfrak{h}_{1}^{2} \rightarrow \mathfrak{h}_{2}^{2} \rightarrow \mathfrak{h}_{2}^{2} / W_{2}$ using $\psi$ for the first arrow. Since $\psi$ induces a homomorphism between the Weyl groups, we can factor the composition to get a map $\mathfrak{h}_{1}^{2} / W_{1} \rightarrow \mathfrak{h}_{2}^{2} / W_{2}$. Finally, consider the composition $\operatorname{Hilb}\left(g_{1}\right) \rightarrow \mathfrak{h}_{1}^{2} / W_{1} \rightarrow \mathfrak{h}_{2}^{2} / W_{2}$ where the first arrow comes from the minimal resolution. This is a continuous map and by the universal property of a minimal resolution, the map lifts to $\operatorname{Hilb}\left(\mathfrak{g}_{1}\right) \rightarrow$ $\operatorname{Hilb}\left(\mathfrak{g}_{2}\right)$.

Let us study this induced map in the case of the reduced Hilbert scheme $\operatorname{Hilb}_{\text {red }}^{n}\left(\mathbb{C}^{2}\right)$, which is a minimal resolution (see appendix A). Take $\psi: \mathfrak{s l}_{m} \rightarrow \mathfrak{s l}_{n}$ inducing a map Hilb $\operatorname{Hid}_{\text {red }}^{m}\left(\mathbb{C}^{2}\right) \rightarrow \operatorname{Hilb}_{\text {red }}^{n}\left(\mathbb{C}^{2}\right)$. In the matrix viewpoint, this map is not given by $[(\psi(A), \psi(B))]$. Consider for example the map $\psi: \mathfrak{s l}_{2} \rightarrow \mathfrak{s l}_{4}$ given on the standard generators $(e, f, h)$ of $\mathfrak{s l}_{2}$ by

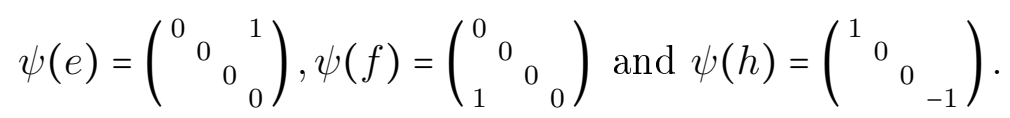


The element $[(h, 0)] \in \operatorname{Hilb}_{\text {red }}^{2}\left(\mathbb{C}^{2}\right)$ corresponds to the ideal $I=\left\langle x^{2}-1, y\right\rangle$ which through $\psi$ goes to $\left\langle x^{4}-x^{2}, y\right\rangle$ which in turn gives the matrices $[(M, 0)]$ where

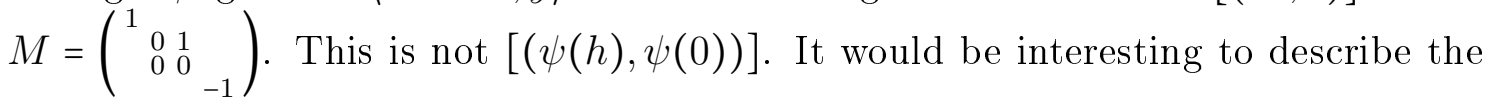
induced map in the matrix viewpoint.

Despite this complication, there are two cases where a map between $\mathfrak{g}$-Hilbert schemes exists naturally.

The first one is linked to the principal map $\psi: \mathfrak{s l}_{2} \rightarrow \mathfrak{g}$ which induces a map

$$
\operatorname{Hilb}\left(\mathfrak{s l}_{2}\right) \rightarrow \operatorname{Hilb}^{\text {reg }}(\mathfrak{g}) .
$$

Indeed, any non-zero element of $\mathfrak{s l}_{2}$ is regular and cyclic. So if $[(A, B)] \in \operatorname{Hilb}\left(\mathfrak{s l}_{2}\right)$ such that $A$ is non-zero, there is by proposition 1.8 a unique representative $(f+t e, B \in$ $Z(e+t f))$ where $(e, f, h)$ denotes the standard generators of $\mathfrak{s l}_{2}$ and $t \in \mathbb{C}$. So the image is $[(\psi(f)+t \psi(e), \psi(B))]$. Since $(\psi(e), \psi(f), \psi(h))$ is a principal $\mathfrak{s l}_{2}$-triple (property of the principal map), we know that $\psi(f)+t \psi(e)$ is in the principal slice, thus it is regular, so we land in $\operatorname{Hilb}^{\text {reg }}(\mathfrak{g})$.

The second one is a sort of inverse map to the first one, but only on the level of the zero-fiber. Given $[(A, B)] \in \operatorname{Hilb}_{0}^{r e g}(\mathfrak{g})$ where $A$ is regular, there is a principal $\mathfrak{s l}_{2}$-subalgebra $\mathcal{S}$ with $A$ as nilpotent element. There is no reason why $B$ should be in $\mathcal{S}$ but there is a "best approximation" in the following sens:

Proposition 1.21. Let $A$ be a principal nilpotent element and $B \in Z(A)$. Then there is a unique $\mu_{2} \in \mathbb{C}$ such that $B-\mu_{2} A$ is not regular.

Proof. The strategy of the proof is to use Proposition B.5 of the appendix which characterizes principal nilpotent elements $x$ as those nilpotent elements whose values $\alpha(x)$ for all simple roots $\alpha$ are non-zero. So the proposition is equivalent to the statement that $\alpha_{1}(B)=\alpha_{2}(B)$ for all simple roots $\alpha_{1}$ and $\alpha_{2}$.

Let $R$ be a root system in $\mathfrak{h}^{*}$ and denote by $R_{+}$and $R_{s}$ the positive and respectively the simple roots. We can conjugate $A$ to the element given by $\alpha(A)=1$ if $\alpha \in R_{s}$ and $\alpha(A)=0$ otherwise.

For two simple roots $\alpha_{1}$ and $\alpha_{2}$ such that $\alpha_{1}+\alpha_{2} \in R$, using $[A, B]=0$ we get:

$$
0=\left(\alpha_{1}+\alpha_{2}\right)([A, B])=\alpha_{1}(A) \alpha_{2}(B)-\alpha_{2}(A) \alpha_{1}(B)=\left(\alpha_{2}-\alpha_{1}\right)(B) .
$$

Since $\mathfrak{g}$ is simple, its Dynkin diagram is connected, so $\alpha_{1}(B)=\alpha_{2}(B)$ for all simple roots. The common value $\mu_{2}$ gives the unique complex number such that $B-\mu_{2} A$ is not regular.

With this proposition, we can now define a map

$$
\mu: \operatorname{Hilb}_{0}^{r e g}(\mathfrak{g}) \rightarrow \operatorname{Hilb}_{0}\left(\mathfrak{s l}_{2}\right)
$$

given by $\mu([(A, B)])=\left[\left(e, \mu_{2} e\right]\right.$ or $\left[\left(\mu_{2} e, e\right)\right]$ depending whether $A$ or $B$ is regular.

An equivalent way to define the map $\mu$ is the following: we can use the previous proposition 1.21 to show that the centralizer $Z(A)$ of a principal nilpotent element is a direct product

$$
Z(A)=\operatorname{Span}(A) \times Z(A)^{i r r e g}
$$

where $Z(A)^{\text {irreg }}$ denotes the irregular elements of $Z(A)$. The map $\mu$ is nothing but the projection to the first factor. 
Remark. We can describe the regular part of the $\mathfrak{g}$-Hilbert scheme $\operatorname{Hilb}^{\text {reg }}(\mathfrak{g})$ as those classes $[(A, B)]$ such that $\operatorname{Span}(A, B)$ intersects the regular part $\mathfrak{g}^{\text {reg }}$ nontrivially. This description is more symmetric since it does not prefer $A$ or $B$. From proposition 1.21 we see that the intersection of $\operatorname{Span}(A, B)$ with $\mathfrak{g}^{\text {reg }}$ is the whole two-dimensional $\operatorname{Span}(A, B)$ from which we have to take out a line. Hence, the intersection has two components.

In the following subsections, we study the regular part $\operatorname{Hilb}^{\text {reg }}(\mathfrak{g})$ and its zero-fiber case by case for classical $\mathfrak{g}$.

1.5. Case $A_{n}$. Consider $\mathfrak{g}=\mathfrak{s l}_{n}$ (of type $A_{n-1}$ ). We describe first $\operatorname{Hilb}_{0}^{\text {reg }}\left(\mathfrak{s l}_{n}\right)$, its idealic map and then $\mathrm{Hilb}^{\text {reg }}\left(\mathfrak{s l}_{n}\right)$ using proposition 1.8 .

Fix the following principal nilpotent element (with 1 on the line just under the main diagonal):

$$
f=\left(\begin{array}{lll}
1 & & \\
& \ddots & \\
& & 1
\end{array}\right) .
$$

This element $f$ is cyclic, so we know from 1.5 that the centralizer is given by polynomials: $Z(f)=\left\{\mu_{2} f+\mu_{3} f^{2}+\ldots+\mu_{n} f^{n-1}\right\}$. So an element of $\operatorname{Hilb}_{0}^{\text {reg }}\left(\mathfrak{s l}_{n}\right)$ can be represented by $(f, Q(f))$ where $Q$ is a polynomial without constant term of degree at most $n-1$. The coefficients $\mu_{i}$ are called higher Beltrami coefficients.

Since here we have $\operatorname{Hilb}_{0}^{\text {reg }}\left(\mathfrak{s l}_{n}\right) \subset \operatorname{Hilb}^{\text {cycl }}\left(\mathfrak{s l}_{n}\right)$ (already $f$ is cyclic), the idealic map is given by

$$
I(f, Q(f))=\{P \in \mathbb{C}[x, y] \mid P(f, Q(f))=0\}=\left\langle x^{n},-y+Q(x)\right\rangle .
$$

We recognize the big cell of the zero-fiber of the punctual Hilbert scheme.

To describe the whole regular part $\operatorname{Hilb}^{\text {reg }}\left(\mathfrak{s l}_{n}\right)$, we take the following principal slice given by companion matrices:

$$
\left(\begin{array}{cccc} 
& & & t_{n} \\
1 & & & \vdots \\
& \ddots & & t_{2} \\
& & 1 &
\end{array}\right) .
$$

Let $A$ be a matrix of companion type. Notice that the characteristic polynomial of a companion matrix is given by $x^{n}+t_{2} x^{n-2}+\ldots+t_{n}$. Since $A$ is still cyclic, its centralizer consists of polynomials in $A$ with constant term determined by the other coefficients (in order to ensure trace zero). Thus, a representative of $\mathrm{Hilb}^{\text {reg }}\left(\mathfrak{s l}_{n}\right)$ is given by $(A, B=Q(A))$.

The idealic map is thus given by

$$
I(A, B)=\left\langle x^{n}+t_{2} x^{n-2}+\ldots+t_{n},-y+\mu_{1}+\mu_{2} x+\ldots+\mu_{n} x^{n-1}\right\rangle
$$

where $\mu_{1}$ is given by $\mu_{1}=\sum_{k=2}^{n-1} \frac{k}{n} t_{k} \mu_{k+1}$. One recognizes the big cell of the reduced punctual Hilbert scheme. Notive that the idealic map is injective here.

1.6. Case $B_{n}$. Consider $\mathfrak{g}=\mathfrak{s o}_{2 n+1}$. Represent $\mathfrak{g}$ on $\mathbb{C}^{2 n+1}$ using the metric given by $g\left(e_{i}, e_{j}\right)=\delta_{i, n-j}$ (where $e_{i}$ are standard vectors), i.e. $g=\left({ }_{1}{ }_{1}^{1}\right)$. A matrix $A$ is in $\mathfrak{g}$ iff $\sigma(A)=-A$ where $\sigma$ is the involution consisting in a reflection along the anti-diagonal. In other words $A \in \mathfrak{g}$ iff $A_{i, j}=A_{n+1-j, n+1-i}$ for all $i, j$. 
We fix the following principal nilpotent element:

$$
f=\left(\begin{array}{llllll}
1 & & & & & \\
& \ddots & & & & \\
& & 1 & & & \\
& & & -1 & & \\
& & & & \ddots & \\
& & & & & -1
\end{array}\right)
$$

This element is cyclic, so its centralizer by 1.5 consists of all odd polynomials: $Z(f)=\left\{\mu_{2} f+\mu_{4} f^{3}+\ldots+\mu_{2 n} f^{2 n-1}\right\}$. A representative of $\operatorname{Hilb}_{0}^{r e g}(\mathfrak{g})$ is thus given by $(f, Q(f))$ where $Q$ is an odd polynomial of degree at most $2 n-1$. The coefficients $\mu_{2 i}$ are called the higher Beltrami coefficients for $B_{n}$.

A principal slice is given by

$$
\left(\begin{array}{ccccccc}
1 & & & & & t_{2 n} & \\
& \ddots & & t_{2} & & \ddots & \\
& & 1 & & -t_{2} & & \\
& & -1 & & & \\
& & & & \ddots & & \\
& & & & & -1 &
\end{array}\right) .
$$

Let $A$ be a matrix of this type. Its characteristic polynomial is given by $x^{2 n+1}-$ $2 t_{2} x^{2 n-1}+2 t_{4} x^{2 n-3} \pm \ldots+(-1)^{n} \times 2 t_{2 n} x$. So we can really think of the principal slice as a generalized companion matrix. Changing slightly $t_{2 i}$ we can get rid of signs and the factor 2 in the characteristic polynomial, which we will do in the sequel.

The matrix $A$ is still cyclic, so we have the inclusion $\operatorname{Hilb}^{\text {reg }}(\mathfrak{g}) \subset \operatorname{Hilb}^{\text {cycl }}(\mathfrak{g})$. A representative of $\operatorname{Hilb}^{\text {reg }}(\mathfrak{g})$ is given by $(A, B=Q(A))$ where $Q$ is still an odd polynomial of degree at most $2 n-1$. The idealic map is then given by

$$
I(A, B)=\left\langle x^{2 n+1}+t_{2} x^{2 n-1}+t_{4} x^{2 n-3}+\ldots+t_{2 n} x,-y+\mu_{2} x+\mu_{4} x^{3}+\ldots+\mu_{2 n} x^{2 n-1}\right\rangle .
$$

This ideal is invariant under the map $(x, y) \mapsto(-x,-y)$. This is not surprising since a generic element of the $\mathfrak{g}$-Hilbert scheme is a pair of two diagonal matrices which for $\mathfrak{s o}_{2 n+1}$ are of the form $\operatorname{diag}\left(x_{1}, \ldots, x_{n}, 0,-x_{n}, \ldots,-x_{1}\right)$ and $\operatorname{diag}\left(y_{1}, \ldots, y_{n}, 0,-y_{n}, \ldots,-y_{1}\right)$. So they can be thought of as $2 n+1$ points in $\mathbb{C}^{2}$ with one point being the origin and the other points being symmetric with respect to the origin. This set is invariant under the map - id, so is its defining ideal.

The next type, $C_{n}$, is quite similar to $B_{n}$.

1.7. Case $C_{n}$. Let $\mathfrak{g}=\mathfrak{s p}_{2 n}$. We use the symplectic structure $\omega=\sum_{i} e_{i} \wedge e_{n+i}$ of $\mathbb{C}^{2 n}$ to represent $\mathfrak{g}$. So a matrix

$$
\left(\begin{array}{l|l}
A & B \\
\hline C & D
\end{array}\right)
$$

is in $\mathfrak{g}$ iff $D=-A^{T}$ and $B$ and $C$ are symmetric matrices.

Fix the principal nilpotent by 


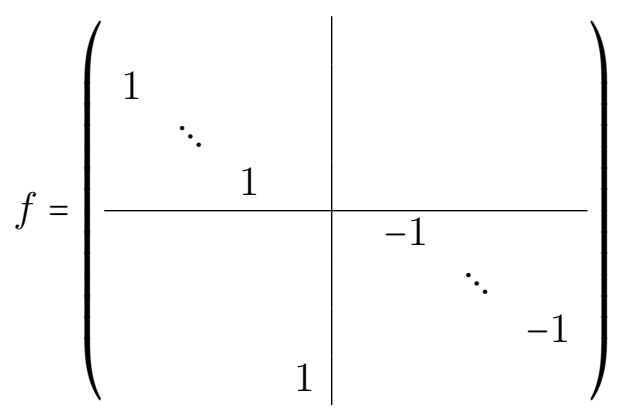

This element is cyclic, so its centralizer is given by odd polynomials: $Z(f)=$ $\left\{\mu_{2} f+\mu_{4} f^{3}+\ldots+\mu_{2 n} f^{2 n-1}\right\}$. As for $B_{n}$ we call the $\mu_{2 i}$ higher Beltrami coefficients.

A principal slice is given by

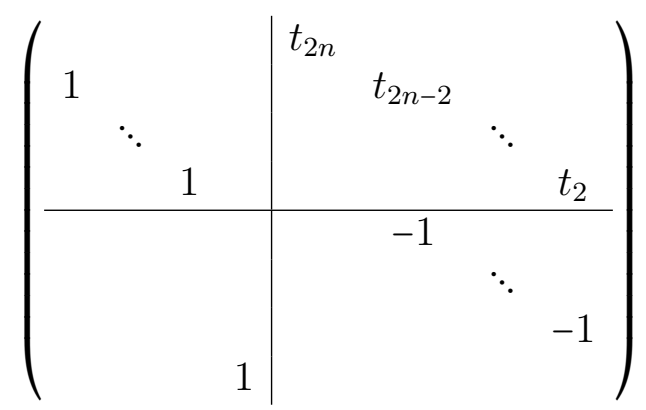

Let $A$ be an element of this form. Its characteristic polynomial is given by $x^{2 n}-$ $t_{2} x^{2 n-2}+t_{4} x^{2 n-4} \pm \ldots+(-1)^{n} t_{2 n}$. By changing signs in the $t_{2 i}$ we can omit the minus signs in the characteristic polynomial.

The matrix $A$ is still cyclic so a representative of $\operatorname{Hilb}^{r e g}\left(\mathfrak{s p}_{2 n}\right)$ is given by $(A, B=$ $Q(A))$ where $Q$ is an odd polynomial of degree at most $2 n-1$.

The idealic map is given by

$$
I(A, B)=\left\langle x^{2 n}+t_{2} x^{2 n-2}+t_{4} x^{2 n-4}+\ldots+t_{2 n},-y+\mu_{2} x+\mu_{4} x^{3}+\ldots+\mu_{2 n} x^{2 n-1}\right\rangle .
$$

As for $B_{n}$, this ideal is invariant under - id which comes from the fact that two diagonal matrices in $\mathfrak{s p}_{2 n}$ give $2 n$ points in $\mathbb{C}^{2}$ which are symmetric with respect to the origin.

The last classical type, $D_{n}$, has some surprises.

1.8. Case $D_{n}$. Let $\mathfrak{g}=\mathfrak{s o}_{2 n}$. We use the same representation as for $B_{n}$.

Fix the following principal nilpotent element:

$$
f=\left(\begin{array}{ccccccc}
1 & & & & & & \\
& \ddots & & & & & \\
& & 1 & & & & \\
& & 1 & 0 & & & \\
& & & -1 & -1 & & \\
& & & & & \ddots & \\
& & & & & & -1
\end{array}\right)
$$

This elements is not cyclic, since $f^{2 n-1}=0$. A direct computation shows that $Z(f)=\left\{\mu_{2} f+\mu_{4} f^{3}+\ldots+\mu_{2 n-2} f^{2 n-3}\right\} \cup\left\{\sigma_{n} S\right\}$ where $S$ is the matrix 


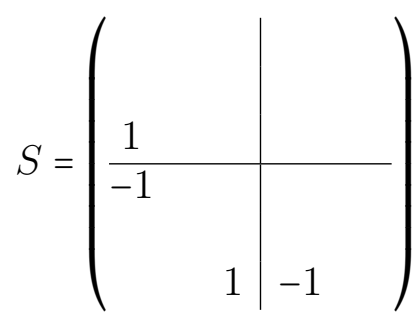

We can give an intrinsic definition of the matrix $S$ : let $R$ be a root system and $v_{\alpha}$ be a root vector in $\mathfrak{g}$ for the root $\alpha \in R$. Choose a base $\alpha_{1}, \ldots, \alpha_{n}$ of $R$ (the simple roots) such that $\alpha_{n-1}$ and $\alpha_{n}$ correspond to the two non-adjacent vertices in the Dynkin diagram of $D_{n}$ (see figure 2). We can choose $f$ to be $\sum_{i} v_{\alpha_{i}}$. The matrix $S$ is then given by

$$
S=v_{\alpha_{1}+\ldots+\alpha_{n-1}} \pm v_{\alpha_{1}+\ldots+\alpha_{n-2}+\alpha_{n}}
$$

where the sign depends on the choice of the root vectors.

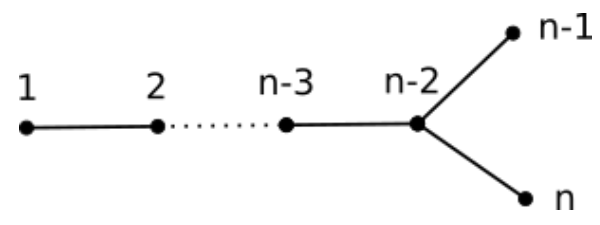

Figure 2. Dynkin diagram for $D_{n}$

A representative of $\operatorname{Hilb}_{0}^{r e g}\left(\mathfrak{s o}_{2 n}\right)$ is given by $\left(A=f, B=Q(f)+\sigma_{n} S\right)$ where $Q$ is an odd polynomial of degree at most $2 n-3$. Such a pair is cyclic iff $\sigma_{n} \neq 0$.

Let us compute the ideal in the cyclic case. One checks easily that $f S=S f$ and that $S^{2}=2 f^{2 n-2}$. Hence for $B=\mu_{2} f+\ldots+\mu_{2 n-2} f^{2 n-2}+\sigma_{n} S$, we get $A B=f B=$ $\mu_{2} f^{2}+\ldots+\mu_{2 n-2} f^{2 n-2}$ and $B^{2}=\left(\mu_{2} f+\ldots+\mu_{2 n-2} f^{2 n-3}\right)^{2}+2 \sigma_{n}^{2} f^{2 n-2}$. Hence, the idealic map is given by

$I(A, B)=\left\langle x^{2 n-1}, x y=\mu_{2} x^{2}+\mu_{4} x^{4}+\ldots+\mu_{2 n-2} x^{2 n-2}, y^{2}=\nu_{2} x^{2}+\nu_{4} x^{4}+\ldots+\nu_{2 n-2} x^{2 n-2}\right\rangle$

where $\nu_{2 k}=\sum_{i=1}^{k} \mu_{2 i} \mu_{2 k+2-2 i}$ for $k=1, \ldots, n-2$ and $\nu_{2 n-2}=2 \sigma_{n}^{2}+\sum_{i=1}^{n-1} \mu_{2 i} \mu_{2 n-2 i}$. So we see that $\left(\mu_{2}, \mu_{4}, \ldots, \mu_{2 n-2}, \nu_{2 n-2}\right)$ is a set of independent variables which we call higher Beltrami differentials for $D_{n}$. We will also call $\sigma_{n}$ a higher Beltrami differential. If $\sigma_{n}=0$, we define the idealic map to be the continuous extension of the above ideal which is still of the same form.

Remark. We have seen in example 1.17 that inside $\operatorname{Hilb}^{\text {cycl }}(\mathfrak{g})$ there is no welldefined continuous extension of the idealic map. But inside the zero-fiber, the limit is unique.

The Hilbert scheme is covered by charts indexed by partitions (see [Ha98]). The chart in which $I$ is written corresponds to the partition $2 n=(2 n-1)+1$ which we write also $[2 n-1,1]$. In fact, this is the highest partition of $2 n$ of type $D_{n}$ (see CM93, chapter 5 for special types of partitions).

A principal slice is given by 


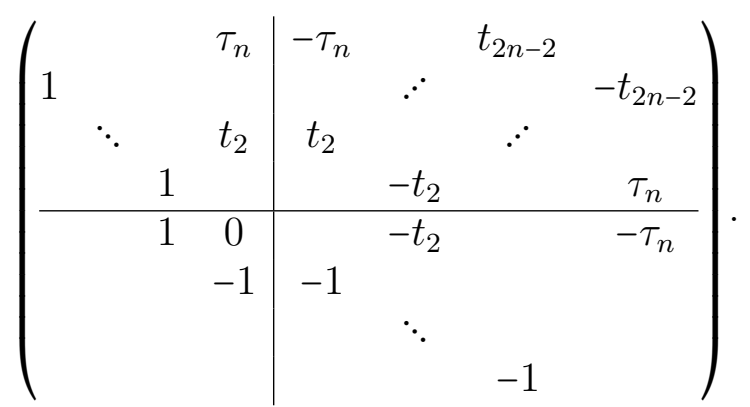

Notice that the matrix for $\tau_{n}$ is $S^{T}$. Let $A$ be a matrix of this type. Its characteristic polynomial is given by

$$
\chi(A)=x^{2 n}-4 t_{2} x^{2 n-2}+4 t_{4} x^{2 n-4} \pm \ldots+(-1)^{n-1} \times 4 t_{2 n-2} x^{2}+(-1)^{n} \tau_{n}^{2} .
$$

By changing signs and factors in $t_{2 i}$ and $\tau_{n}$, we can omit signs and the factor 4 in the characteristic polynomial.

One can compute that the minimal polynomial of $A$ is equal to the characteristic polynomial iff $\tau_{n} \neq 0$. So $A$ is cyclic iff $\tau_{n} \neq 0$ (by proposition B.3). In that case, the centralizer consists of all odd polynomials in $A$ of degree at most $2 n-1$. If $\tau_{n}=0$, the centralizer is given by

$$
Z(A)=\left\{\mu_{2} A+\mu_{4} A^{3}+\ldots+\mu_{2 n-2} A^{2 n-3}\right\} \cup\left\{\sigma_{n} S_{t}\right\}
$$

where the matrix $S_{t}$ is given by $S_{t}=S+t_{2 n-2} S^{T}$. The minimal polynomial is given by $\chi(x) / x$ (which is a polynomial since $\tau_{n}=0$ ).

The pair $(A, B)$ is cyclic iff either $\tau_{n} \neq 0$ or $\tau_{n}=0$ and $\sigma_{n} \neq 0$. In the first case, the idealic map is given by

$$
I=\left\langle x^{2 n}+t_{2} x^{2 n-2}+t_{4} x^{2 n-4}+\ldots+t_{2 n-2} x^{2}+\tau_{n}^{2},-y+\mu_{2} x+\mu_{4} x^{3}+\ldots+\mu_{2 n} x^{2 n-1}\right\rangle .
$$

In the second case, we need three generators for the ideal, like for the zero-fiber. We can compute that

$$
\begin{aligned}
I(A, B)=\left\langle x^{2 n-1}\right. & =u_{2} x+u_{4} x^{3}+\ldots+u_{2 n-2} x^{2 n-3}+u y, \\
x y & =v_{0}+v_{2} x^{2}+\ldots+v_{2 n-2} x^{2 n-2}, \\
y^{2} & \left.=w_{0}+w_{2} x^{2}+\ldots+w_{2 n-2} x^{2 n-2}\right\rangle
\end{aligned}
$$

where the coordinates can be chosen to be $\left(u_{2}, u_{4}, \ldots, u_{2 n-2}, u, v_{2}, \ldots, v_{2 n-2}, w_{2 n-2}\right)$, i.e. all the other variables are functions of these. For a unified way to get coordinates in Hilbert schemes, see subsection 3.5] or directly Haiman's paper [Ha98].

The second ideal is in the chart corresponding to the partition $[2 n-1,1]$ whereas the first corresponds to the trivial partition [2n]. If $u \neq 0$ we can write the second ideal in the first chart, i.e. perform a coordinate change in the Hilbert scheme. The link between the coordinates is given by

$$
\left\{\begin{array}{cc}
\tau_{n}^{2}=u v_{0} & \\
\mu_{2 n}=\frac{1}{u} & \\
\mu_{2 k}=-\frac{u_{2 k}}{u} & \text { for } 1 \leq k<n \\
t_{2 k}=u_{2 n-2 k}+u v_{2 n-2 k} & \text { for } 1 \leq k \leq n-1
\end{array}\right.
$$

A regular pair $[(A, B)]$ which is not cyclic has both $\tau_{n}$ and $\sigma_{n}$ equal to 0 . In that case, we define the idealic map $I(A, B)$ to be the limit of $I\left(A, B+t S_{t}\right)$ for $t \in \mathbb{C}$ goes to 0 . So we stay in a chart associated to the partition $[2 n-1,1]$. 
Notice that the map from Hilb ${ }^{r e g}(\mathfrak{g})$ to the space of ideals $I_{\mathfrak{g}}\left(\mathbb{C}^{2}\right)$ is not injective, since for $\tau_{n}$ and $-\tau_{n}$ we get the same ideal. Even in the zero-fiber the map is not injective, since $\sigma_{n}$ and $-\sigma_{n}$ give the same ideal. In addition, the map is not surjective neither. Indeed the ideal $I=\left\langle x^{5}-y, x y, y^{2}\right\rangle \in I_{\mathfrak{g}}\left(\mathbb{C}^{2}\right)$ is not in the image since with the notations above we have $v_{0}=0$ and $u \neq 0$. Changing the chart, we can compute that $\tau_{n}^{2}=u v_{0}=0$. But for a matrix in $\operatorname{Hilb}^{r e g}(\mathfrak{g})$ with $\tau_{n}=0$ we get $u=0$.

Remark. In the usual Hilbert scheme, there is only one cell of maximal dimension. Comparing type $C_{n}$ and type $D_{n}$, we see that the zero-fiber of

$$
\{I \text { ideal of } \mathbb{C}[x, y] \mid \operatorname{codim} I=2 n, I \text { invariant under }-\mathrm{id}\}
$$

has two components of maximal dimension, those corresponding to the zero-fibers $\operatorname{Hilb}_{0}^{r e g}\left(\mathfrak{s p}_{2 n}\right)$ and $\operatorname{Hilb}_{0}^{\text {reg }}\left(\mathfrak{s o}_{2 n}\right)$.

Remark. We notice the following analogue to Higgs bundles: the pair $[(f, 0)] \epsilon$ $\operatorname{Hilb}\left(\mathfrak{s o}_{2 n}\right)$ corresponds to the Higgs field given by $\Phi=f$ on the bundle $V=K^{2} \oplus K \oplus$ $K^{0} \oplus K^{-2} \oplus K^{-1} \oplus K^{0}$. This Higgs bundle $(V, \Phi)$ is not stable, only polystable. This could explain why the idealic map can not be continuously extended to $[(f, 0)]$, but the link between Higgs bundles and higher complex structures remains unclear, see also the perspective given in section 4.

1.9. Topology of $\mathfrak{g}$-Hilbert schemes. It is clear that $\operatorname{Hilb}(\mathfrak{g})$ is a topological space, as a quotient of a subset of $\mathfrak{g}^{2}$. In this section, we explore this topology of $\operatorname{Hilb}(\mathfrak{g})$, especially for $\mathfrak{g}=\mathfrak{s l}_{n}$. We then formulate some conjectures on its general structure.

For $\mathfrak{g}=\mathfrak{s l}_{2}$, every non-zero element $A \in \mathfrak{g}$ is regular and cyclic. Since the centralizer of the pair $(0,0)$ is all of $\mathfrak{s l}_{2}$, this pair is not in $\operatorname{Hilb}\left(\mathfrak{s l}_{2}\right)$. Thus we have $\operatorname{Hilb}\left(\mathfrak{s l}_{2}\right)=$ $\operatorname{Hilb}^{\text {cycl }}\left(\mathfrak{s l}_{2}\right)=\mathrm{Hilb}_{\text {red }}^{2}\left(\mathbb{C}^{2}\right)$ which is a smooth projective variety.

For $\mathfrak{g}=\mathfrak{s l}_{3}$, a detailed analysis, putting $A$ into Jordan normal form, shows that $(A, B)$ has minimal centralizer and is not cyclic iff it is conjugated to a pair $P_{1}(b):=$ $\left(\left(\begin{array}{lll}0 & 1 & 0 \\ 0 & 0 & 0 \\ 0 & 0 & 0\end{array}\right),\left(\begin{array}{lll}0 & b & 1 \\ 0 & 0 & 0 \\ 0 & 0 & 0\end{array}\right)\right)$. So

$$
\operatorname{Hilb}\left(\mathfrak{s l}_{3}\right)=\operatorname{Hilb}_{\text {red }}^{3}\left(\mathbb{C}^{2}\right) \cup\left\{P_{1}(b) \mid b \in \mathbb{C}\right\} .
$$

At first sight, the topology seems to be a smooth variety (the reduced Hilbert scheme) and a complex line. But a closer look shows that each point of the extra line is infinitesimally close to a point in the variety, meaning that these two points cannot be separated by open sets, infringing the Hausdorff property. The pair $P_{1}(b)$ is infinitesimally close to $P_{2}(b):=\left(\left(\begin{array}{lll}0 & 1 & 0 \\ 0 & 0 & 0 \\ 0 & 0 & 0\end{array}\right),\left(\begin{array}{lll}0 & 0 & 0 \\ 0 & 0 & 0 \\ 0 & 1 & 0\end{array}\right)\right)$. Indeed any neighborhood of the first pair $P_{1}(b)$ contains $\left(\left(\begin{array}{lll}0 & 1 & 0 \\ 0 & 0 & 0 \\ 0 & 0 & 0\end{array}\right),\left(\begin{array}{lll}0 & b & 1 \\ 0 & 0 & 0 \\ 0 & s & 0\end{array}\right)\right)$ for some small $s \in \mathbb{C}$ which is conjugated to $\left(\left(\begin{array}{lll}0 & 1 & 0 \\ 0 & 0 & 0 \\ 0 & 0 & 0\end{array}\right),\left(\begin{array}{lll}0 & b & s \\ 0 & 0 & 0 \\ 0 & 1 & 0\end{array}\right)\right)$ which lies in a neighborhood of the second pair $P_{2}(b)$. Since the idealic map is continuous and for $\mathfrak{s l}_{n}$ injective on the cyclic part, there cannot be another point of the cyclic part which is infinitesimally close to the first pair $P_{1}(b)$. Finally, two elements of the extra line can be separated by open sets. Hence, the space $\operatorname{Hilb}\left(\mathfrak{s l}_{3}\right)$ is obtained from a smooth variety by adding "double points" (here in the sens of infinitesimally close points) along a complex line.

Since the idealic map is injective on the cyclic part $\operatorname{Hilb}^{c y c l}\left(\mathfrak{s l}_{n}\right)$, the same analysis holds for $\mathfrak{s l}_{n}$, i.e. Hilb $\left(\mathfrak{s l}_{n}\right)$ is obtained from a smooth variety (the reduced Hilbert scheme) by adding double points. 
There should exist a procedure, like a GIT quotient, giving a modified $\mathfrak{g}$-Hilbert scheme which is a Hausdorff space. The GIT quotient does not apply here since $\left\{(A, B) \in \mathfrak{g}^{2} \mid[A, B]=0, \operatorname{dim} Z(A, B)=\operatorname{rk} \mathfrak{g}\right\}$ is not a closed variety. In the language of GIT quotients, the pairs $P_{1}$ and $P_{2}$ above are both semistable, but there is no polystable element in their closure.

To give a feeling on what happens, consider the action of $\mathbb{R}_{>0}$ on $\mathbb{R}^{2} \backslash\{(0,0)\}$ given by $\lambda$. $\left(x_{1}, x_{2}\right)=\left(\lambda x_{1}, \lambda^{-1} x_{2}\right)$. The orbits are drawn in figure 3 . The quotient space is a set of two lines $L_{1}$ and $L_{2}$ with origins $O_{1}$ and $O_{2}$ together with two extra points $O_{3}$ and $O_{4}$ such that the pairs $\left(O_{1}, O_{3}\right),\left(O_{1}, O_{4}\right),\left(O_{2}, O_{3}\right)$ and $\left(O_{2}, O_{4}\right)$ are infinitesimally close points (the four points $O_{i}$ correspond to the four half-axis). In the figure, the dashed lines indicate infinitesimally close points. From the GIT perspective, all points are semistable (take the constant function 1), the four halfaxis are semistable and all other orbits are stable. The orbits of the half-axis are closed in $\mathbb{R}^{2} \backslash\{(0,0)\}$ so they should be polystable, but in the quotient the points are still infinitesimally close.
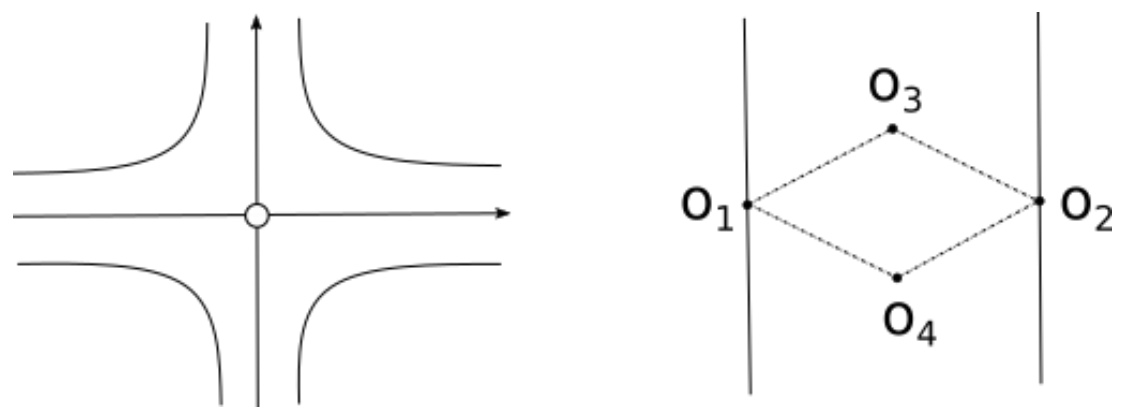

FiguRE 3. Non-Hausdorff quotient

We conjecture the following:

Conjecture 1.22. There is a generalized GIT quotient procedure identifying infinitesimally close points in $\operatorname{Hilb}(\mathfrak{g})$, giving a modified $\mathfrak{g}$-Hilbert scheme which is Hausdorff.

In particular one should find the reduced Hilbert scheme for $\mathfrak{g}=\mathfrak{s l}_{n}$. See also conjecture 1.16 for a modified $\mathfrak{g}$-Hilbert scheme as a resolution of $\mathfrak{h}^{2} / W$.

Assume a smooth version of the $\mathfrak{g}$-Hilbert scheme exists. In the $\mathfrak{s l}_{n}$-case the reduced Hilbert scheme is covered by charts parametrized by partitions of $n$, which also parametrizes nilpotent orbits of $\mathfrak{s l}_{n}$. For $\mathfrak{g}$ of classical type, the nilpotent orbits are parametrized by special partitions (see [CM93], chapter 5). In general, we conjecture the following for the zero-fiber of the $\mathfrak{g}$-Hilbert scheme:

Conjecture 1.23. The smooth version of $\operatorname{Hilb}_{0}(\mathfrak{g})$ is covered by charts parametrized by nilpotent orbits and all these charts are necessary to cover $\operatorname{Hilb}_{0}(\mathfrak{g})$. In particular for classical $\mathfrak{g}$, we conjecture that the modified version of $\mathrm{Hilb}_{0}(\mathfrak{g})$ is isomorphic to the space of ideals of $\mathbb{C}[x, y]$ which are of codimension $m, W$-invariant, supported at 0 and which lie in a chart associated to a partition of type $\mathfrak{g}$.

In particular, for every nilpotent $A \in \mathfrak{g}$, there has to be an element in $\operatorname{Hilb}(\mathfrak{g})$ containing the conjugacy class of $A$. More precisely, we conjecture: 
Conjecture 1.24. Let $\mathfrak{g}$ be of rank at least 3. For a nilpotent element $A \in \mathfrak{g}$, there is $B \in Z(A)$ nilpotent such that $\operatorname{dim} Z(A, B)=\operatorname{rk} \mathfrak{g}$, i.e. $[(A, B)] \in \operatorname{Hilb}_{0}(\mathfrak{g})$. We conjecture that this should be true for a generic element $B \in Z(A)$.

For $\mathfrak{s l}_{n}$ the conjecture is true: we can associate to a nilpotent element $A$ a partition $\nu$. To the transpose partition $\nu^{T}$ (using the transpose of the Young diagram) correspond a nilpotent element $B$ which satisfies the requirements since $(A, B)$ is cyclic. An equivalent way is to use example 1.3 to produce $B$.

For $\mathfrak{g}=\mathfrak{s p}_{4}$ of type $C_{2}$, there is the following counterexample. That is why we formulate the conjecture only for Lie algebras of rank at least 3. Take the nilpotent element

$$
A=\left(\begin{array}{c|c}
0 & \text { id } \\
\hline 0 & 0
\end{array}\right) .
$$

Its centralizer is given by

$$
Z(A)=\left(\begin{array}{cc|cc}
0 & b & x & y \\
-b & 0 & y & z \\
\hline 0 & 0 & 0 & b \\
0 & 0 & -b & 0
\end{array}\right) .
$$

An element $B$ of the centralizer is nilpotent iff $b=0$. In that case the common centralizer $Z(A, B)$ is at least of dimension 3 , so $[(A, B)]$ is not in $\operatorname{Hilb}_{0}\left(\mathfrak{s p}_{4}\right)$.

In general, we cannot hope to find $B \in Z(A)$ such that $(A, B)$ is cyclic. For example take $\mathfrak{g}=\mathfrak{s p}_{16}$ and $A$ a nilpotent element corresponding to the partition $[7,5,3,1]$ of 16 . If there is $B \in Z(A)$ nilpotent and such that $(A, B)$ is cyclic, there would be an ideal $I$ of codimension 16 whose associated matrices are $A$ and $B$ (see example 1.3). Using $A$, we see that $I$ has to be of the form

$$
I=\left\langle x^{4}, x^{3} y, x^{2} y^{3}, x y^{5}, y^{7}=Q(x, y)\right\rangle
$$

where $Q$ is a polynomial with monomial terms in the Young diagram $D$. A partition of type $C_{n}$ has all odd parts with even multiplicity and one can check that for all choices of the polynomial $Q$, the ideal $I$ is never in a chart with all odd parts with even multiplicity.

\section{2. $\mathfrak{g}$-COMPLEX STRUCTURES}

Using the $\mathfrak{g}$-Hilbert scheme we are able to construct a new geometric structure on a smooth surface, generalizing complex structures and higher complex structures. The construction and methods are inspired by those used for higher complex structures in FT19. We recall the ideas of constructing higher complex structures before defining the $\mathfrak{g}$-complex structure.

2.1. Complex and higher complex structures. A complex structure on a surface $\Sigma$ is completely encoded in the Beltrami differential.

This goes as follows: For surfaces, a complex structure is equivalent to an almost complex structure, i.e. an endomorphism $J(z)$ in $T_{z}^{*} \Sigma$ such that $J^{2}=-$ id and varying smoothly with $z \in \Sigma(J$ imitates the multiplication by $i$ ). We can diagonalize $J$ by complexifying the cotangent bundle. We get a decomposition into eigenspaces $T^{* \mathbb{C}} \Sigma=T^{*(1,0)} \Sigma \oplus T^{*(0,1)} \Sigma$. In addition $T^{*(1,0)} \Sigma$ is the complex conjugate of $T^{*(0,1)} \Sigma$, so one determines the other. Hence, the complex structure is completely encoded in a direction in each complexified cotangent space, i.e. in a section $s$ of $\mathbb{P}\left(T^{*} \mathbb{C} \Sigma\right)$ which is 
nowhere real (meaning $s$ and $\bar{s}$ are linear independent). The projectivization can also be obtained by the zero-fiber of the punctual Hilbert scheme of length 2 : $\operatorname{Hilb}_{0}^{2}\left(\mathbb{C}^{2}\right) \cong$ $\mathbb{P}\left(\mathbb{C}^{2}\right)$. In coordinates, we can write $T_{z}^{*(0,1)} \Sigma=\operatorname{Span}\left(\bar{p}-\mu_{2}(z) p\right)$ where $p$ and $\bar{p}$ are linear coordinates on $T^{* \mathbb{C}} \Sigma$. The coefficient $\mu_{2}(z)$ is the Beltrami differential. The condition that the section $s$ is nowhere real translates to $\mu_{2}(z) \bar{\mu}_{2}(z) \neq 1$ for all $z \in \Sigma$.

Generalizing this idea, we defined in [FT19] the higher complex structure as a section $I$ of $\operatorname{Hilb}_{0}^{n}\left(T^{* \mathbb{C}} \Sigma\right)$ satisfying $I(z) \oplus \bar{I}(z)=\langle p, \bar{p}\rangle$ at every point $z \in \Sigma$. Here $p$ and $\bar{p}$ are linear coordinates on $T^{* \mathbb{C}} \sum$. The condition on $I$ generalizes the condition above of a nowhere real section. We call it the reality constraint.

We use exclusively the idealic viewpoint of the punctual Hilbert scheme in this definition. Since the $\mathfrak{g}$-Hilbert scheme uses the matrix viewpoint, we have to rewrite the definition of higher complex structure in that picture. So we replace the ideal $I(z)$ by a conjugacy class of commuting matrices $A(z)$ and $B(z)$. We can put them together in a gauge class of a $\mathfrak{s l}_{n}$-valued 1-form $\Phi(z)=A(a) d z+B(z) d \bar{z}$. The commutativity of $A$ and $B$ translates to the fact that $\Phi$ satisfies $\Phi \wedge \Phi=0$.

It is not surprising to use 1-forms since a generic point of the Hilbert scheme gives $n$ distinct points in each fiber $T_{z}^{* \mathbb{C}} \Sigma$ which can be put together to $n$ sections of $T^{* \mathbb{C}} \Sigma$, i.e. a $n$-tuple of complex 1-forms. Going to the zero-fiber of the Hilbert scheme means that all these 1-forms are collapsed to the zero-section $\Sigma \subset T^{* \mathbb{C}} \Sigma$.

2.2. Definition. We are now ready to give the definition of a $\mathfrak{g}$-complex structure, but one difficulty stays: we have to incorporate the reality constraint in the matrix viewpoint. Recall from 1.4 equation $(1.3)$ the map $\mu_{2}: \mathrm{Hilb}_{0}^{\text {reg }}(\mathfrak{g}) \rightarrow \mathbb{C}$ associating to $[(A, B)]$ the unique $\mu_{2} \in \mathbb{C}$ such that $B-\mu_{2} A$ is irregular.

Definition 2.1. A $\mathfrak{g}$-complex structure is a gauge class of elements

$$
A(z) d z+B(z) d \bar{z} \in \Omega^{1}(\Sigma, \mathfrak{g})=\Omega^{1}(\Sigma, \mathbb{C}) \otimes \mathfrak{g}
$$

such that

$$
[(A(z), B(z))] \in \operatorname{Hilb}_{0}^{r e g}(\mathfrak{g})
$$

and $\mu_{2}(z) \bar{\mu}_{2}(z) \neq 1$ for all $z \in \Sigma$.

Notice that for complex structures, the map $\mu_{2}(z)$ is nothing but the Beltrami differential. So our reality constraint coincides with the one for complex structures. In particular, for $\mathfrak{g}=\mathfrak{s l}_{2}$, we get a usual complex structure. In the general case, we have the following:

Proposition 2.2. A $\mathfrak{g}$-complex structure induces a complex structure on $\Sigma$.

Proof. Recall from 1.4 equation (1.3) the map $\mu: \operatorname{Hilb}_{0}^{r e g}(\mathfrak{g}) \rightarrow \operatorname{Hilb}_{0}\left(\mathfrak{s l}_{2}\right)$ given by $\mu([(A, B)])=\left[\left(e, \mu_{2} e\right)\right]$ or $\left[\left(\mu_{2} e, e\right)\right]$ depending on whether $A$ or $B$ is regular. Since a $\mathfrak{s l}_{2}$-complex structure is a complex structure, the map $\mu$ induces a map from $\mathfrak{g}$-complex structures to complex structures.

Remark. To define the map $\mu$ in 1.4, we really need $\mathfrak{g}$ to be simple. Thus, we only get a unique complex structure out of a $\mathfrak{g}$-complex structure for $\mathfrak{g}$ simple.

In the definition of a higher complex structure in [FT19, we use the zero-fiber $\operatorname{Hilb}_{0}^{n}\left(\mathbb{C}^{2}\right)$, without imposing to be in the regular part. The fact that we actually are in the regular part follows from the reality constraint $I \oplus \bar{I}=\langle p, \bar{p}\rangle$. The same can be obtained for $\mathfrak{g}$ of classical type, where we can reformulate the definition of $\mathfrak{g}$-complex structures in a nicer way using the idealic map. 
2.3. Idealic viewpoint. Recall the space of ideals $I_{\mathfrak{g}}\left(\mathbb{C}^{2}\right)$ constructed in 1.3 . Denote by $I_{\mathfrak{g}, 0}\left(\mathbb{C}^{2}\right)$ the set of those ideals of $I_{\mathfrak{g}}\left(\mathbb{C}^{2}\right)$ which are supported on the origin (the zero-fiber). We can rewrite the definition of a $\mathfrak{g}$-complex structure in the following way:

Definition 2.3. For classical $\mathfrak{g}$, a $\mathfrak{g}$-complex structure is a section I of $I_{\mathfrak{g}, 0}\left(T^{* \mathbb{C}} \Sigma\right)$ such that

$$
I(z) \oplus \bar{I}(z)= \begin{cases}\langle p, \bar{p}\rangle & \text { if } \mathfrak{g} \text { of type } A_{n}, B_{n}, C_{n} \\ \langle p, \bar{p}\rangle^{2} & \text { if } \mathfrak{g} \text { of type } D_{n} .\end{cases}
$$

Notice that the condition on the ideals does not depend on coordinates since $\langle p, \bar{p}\rangle$ is the maximal ideal associated to the origin.

We prove the equivalence of both definitions. For that recall that to an ideal $I$ one can associate a class of commuting matrices $[(A, B)]$ (see A.2.).

Proposition 2.4. For classical $\mathfrak{g}$, the condition on $I \oplus \bar{I}$ given in definition 2.3 is equivalent to $[(A(z), B(z))]$ being in the regular part $\operatorname{Hilb}_{0}^{\text {reg }}(\mathfrak{g})$ and having $\mu_{2} \bar{\mu}_{2} \neq 1$, i.e. the condition in definition 2.1.

Proof. The backwards direction is a direct computation using the preferred representatives for $\operatorname{Hilb}_{0}^{r e g}(\mathfrak{g})$ from proposition 1.8. So we concentrate on the direct implication.

Case $A_{n}$. The case $\mathfrak{g}$ of type $A_{n}$ has been treated in [FT19, appendix 5.1. The idea of the proof is similar to the case $D_{n}$ below.

Case $B_{n}$. For $\mathfrak{g}$ of type $B_{n}$ the standard representation gives $\mathfrak{s o}_{2 n+1} \hookrightarrow \mathfrak{s l}_{2 n+1}$. By virtue of the case $A_{n}$, we know that $I \oplus \bar{I}=\langle p, \bar{p}\rangle$ implies $\mu_{2} \bar{\mu}_{2} \neq 1$ and $(A, B)$ regular for $\mathfrak{s l}_{2 n+1}$, i.e.

$$
I(A, B)=\left\langle p^{2 n+1},-\bar{p}+\mu_{2} p+\mu_{3} p^{2}+\ldots+\mu_{2 n} p^{2 n}\right\rangle .
$$

Since we know that in case $B_{n}$, the ideal $I$ is invariant under the map -id, we get $\mu_{2 k+1}=0$ for all $k=1, \ldots, n-1$. So $I$ corresponds to a pair $(f, Q(f))$ for $Q$ an odd polynomial of degree at most $2 n-1$, which is precisely a representative of $\operatorname{Hilb}_{0}^{\text {reg }}\left(\mathfrak{s o}_{2 n+1}\right)$ (see subsection 1.6).

Case $C_{n}$. This case is exactly analogous to $B_{n}$ via the injection $\mathfrak{s p}_{2 n} \hookrightarrow \mathfrak{s l}_{2 n}$.

Case $D_{n}$. We imitate the strategy of the proof for case $A_{n}$ in [FT19] appendix 5.1 with only difference that we have to go further in the analysis, needing some computations. The main argument is an iteration process which always ends since $p^{k} \bar{p}^{l}=0 \bmod I$ for $k+l \geq 2 n$.

Put $I_{1}=\left(I \bmod \langle p, \bar{p}\rangle^{2}\right)$, i.e. the set of all terms of degree at most 1 appearing in $I$. If $I_{1}$ is of dimension 2 , then $I=\langle p, \bar{p}\rangle$ since both $p$ and $\bar{p}$ can be expressed by higher terms which by iteration become 0 . If $I_{1}$ is of dimension 1 , then we have a relation of the form $\bar{p}=\mu_{2} p+p^{2} R(p, \bar{p})$ where $R$ is a polynomial, which gives $\bar{p}$ as a polynomial in $p$ by iteration. We can then explicitly check that $I \oplus \bar{I}$ is either $\langle p, \bar{p}\rangle$ or $\left\langle p=\bar{p}, p \bar{p}, p^{2}\right\rangle$. Hence $I_{1}=\{0\}$.

Put $I_{2}=\left(I \bmod \langle p, \bar{p}\rangle^{3}\right)$. We have $I_{2} \oplus \bar{I}_{2}=(I \oplus \bar{I})_{2}=\left\langle p^{2}, p \bar{p}, \bar{p}^{2}\right\rangle$ by assumption on $I$. If $I_{2}$ is of dimension 3 , then all of $p^{2}, p \bar{p}$ and $\bar{p}^{2}$ can be expressed by higher terms. By iteration, we get $I=\left\langle p^{2}, p \bar{p}, \bar{p}^{2}\right\rangle$ which is not of type $D_{n}$. If $\operatorname{dim} I_{2} \leq 1$, then we also have $\operatorname{dim} \bar{I}_{2} \leq 1$, so $2 \geq \operatorname{dim} I_{2}+\operatorname{dim} \bar{I}_{2}=\operatorname{dim}\left\langle p^{2}, p \bar{p}, \bar{p}^{2}\right\rangle_{2}=3$, a contradiction. Hence $\operatorname{dim} I_{2}=2$.

There is a term containing $p \bar{p}$ in $I_{2}$ since if not, no such term would neither exist in $\bar{I}_{2}$, so neither in $I_{2} \oplus \bar{I}_{2}=\left\langle p^{2}, p \bar{p}, \bar{p}^{2}\right\rangle$, a contradiction. Without loss of generality, 
we can assume that there is another term containing $\bar{p}^{2}$ (if not change the role of $I$ and $\bar{I}$ ).

So there exist $\alpha, \beta, \gamma, \delta \in \mathbb{C}$ such that

$$
\begin{cases}\bar{p}^{2}=\alpha p^{2}+\beta p \bar{p} & \bmod I_{2} \\ p \bar{p}=\gamma p^{2}+\delta \bar{p}^{2} & \bmod I_{2}\end{cases}
$$

If $\beta \gamma \neq 1$, we can simplify by substitution one into the other to

$$
\begin{cases}\bar{p}^{2}=\alpha^{\prime} p^{2} & \bmod I_{2} \\ p \bar{p}=\gamma^{\prime} p^{2} & \bmod I_{2}\end{cases}
$$

If $\beta \gamma=1$, we have $p^{2} \in I_{2}$, so $p \bar{p}=\delta \bar{p}^{2} \bmod I_{2}$, so changing $I$ to $\bar{I}$ we are in the previous situation.

Iterating the substitution process we get that $\bar{p}^{2}$ and $p \bar{p}$ are polynomials in $p$. Using the invariance of $I$ under - id, we see that these are polynomials in $p^{2}$, i.e. even polynomials. So the most generic ideal is given by

$$
I=\left\langle p^{2 n-1}, p \bar{p}=\mu_{2} p^{2}+\mu_{4} p^{4}+\ldots+\mu_{2 n-2} p^{2 n-2}, \bar{p}^{2}=\nu_{2} p^{2}+\nu_{4} p^{4}+\ldots+\nu_{2 n-2} p^{2 n-2}\right\rangle
$$

which corresponds to a regular element of $\operatorname{Hilb}_{0}^{r e g}\left(\mathfrak{s o}_{2 n}\right)$. One checks that $I \oplus \bar{I}$ with $I$ of the form above equals $\langle p, \bar{p}\rangle^{2}$ iff $\mu_{2} \overline{\mu_{2}} \neq 1$.

To end this section, we determine the geometric nature of the various higher Beltrami coefficients. Since $p$ and $\bar{p}$ are linear coordinates on $T^{* \mathbb{C}} \Sigma$, we can identify $p=\frac{\partial}{\partial z}=\partial$ and $\bar{p}=\frac{\partial}{\partial \bar{z}}=\bar{\partial}$. Denote by $K$ the canonical bundle, i.e. $K=T^{*(1,0)} \Sigma$, and by $\Gamma(B)$ the space of sections of a bundle $B$.

Analyzing the behavior under a coordinate change $z \mapsto w(z, \bar{z})$ analogous to the computation in [FT19] section 3.1., we get

$$
\mu_{i} \in \Gamma\left(K^{1-i} \otimes \bar{K}\right) \text { and } \nu_{2 i} \in \Gamma\left(K^{-2 i} \otimes \bar{K}^{2}\right) .
$$

Since $\sigma_{n}^{2}$ has the same nature as $\nu_{2 n-2}$, we get $\sigma_{n} \in \Gamma\left(K^{1-n} \otimes \bar{K}\right)$.

\section{Moduli SPACE}

In this section, we define the moduli space of $\mathfrak{g}$-complex structures and explore its properties. In the whole section $\mathfrak{g}$ is of classical type. We first have to define an equivalence relation on $\mathfrak{g}$-complex structures, which is accomplished by the notion of higher diffeomorphisms.

3.1. Higher diffeomorphisms. In order to get a finite-dimensional moduli space, it is not sufficient to quotient by the diffeomorphisms of $\Sigma$ isotopic to the identity, as for Teichmüller space. The reason is that the $\mathfrak{g}$-complex structure is non-linear in the cotangent spaces $T_{z}^{\star \mathbb{C}} \Sigma$. Diffeomorphisms act linearly on the cotangent space, so it cannot act much on $\mathfrak{g}$-complex structures.

For higher complex structures, in [FT19] section 3.2 we defined higher diffeomorphisms to be Hamiltonian diffeomorphisms of $T^{*} \Sigma$ preserving the zero-section $\Sigma \subset T^{*} \Sigma$. This gives the higher diffeomorphisms for type $A_{n}$. We generalize this idea to other classical $\mathfrak{g}$. Recall the standard representation of $\mathfrak{g}$ on $\mathbb{C}^{m}$ (i.e. $\mathfrak{s l}_{n} \subset \mathfrak{g l}_{n}, \mathfrak{s o}_{n} \subset \mathfrak{g l}_{n}$ and $\left.\mathfrak{s p}_{2 n} \subset \mathfrak{g l}_{2 n}\right)$.

As stated several times, one should think of a $\mathfrak{g}$-complex structure as a $m$-tuple of 1 -forms with some symmetry, which collapses all to the zero-section. The space of higher diffeomorphisms which we are looking for has to preserve this symmetry. For example for $\mathfrak{s l}_{n}$, we have $n$ sections whose barycenter at every fiber is the origin, 
i.e. their sum gives the zero-section. That is why we have to impose that the Hamiltonian diffeomorphisms of $T^{*} \Sigma$ have to preserve the zero-section.

For $\mathfrak{g}$ of type $B_{n}, C_{n}$ or $D_{n}$, the set of $m$ points is symmetric with respect to the origin. Thus we define:

Definition 3.1. A higher diffeomorphism of type $B_{n}, C_{n}$ or $D_{n}$ is a Hamiltonian diffeomorphism of $T^{*} \Sigma$ invariant under the map $(z, p, \bar{p}) \mapsto(z,-p,-\bar{p})$. We denote by $\operatorname{Symp}(\mathfrak{g}, \Sigma)$ the space of higher diffeomorphisms of type $\mathfrak{g}$.

In coordinates a Hamiltonian diffeomorphism is generated by a function $H(z, \bar{z}, p, \bar{p})$ which can be Taylor developed to $\sum_{k, l} w_{k, l}(z, \bar{z}) p^{k} \bar{p}^{l}$. The associated flow preserves the zero-section iff $w_{0,0}=0$. It is invariant under -id iff it has only odd terms, i.e. $w_{k, l}=0$ for all $k+l$ even.

3.2. Action on $\mathfrak{g}$-complex structures. We can now analyze how higher diffeomorphisms act on $\mathfrak{g}$-complex structures.

Intuitively, Hamiltonian diffeomorphisms of $T^{*} \Sigma$ act on the space of 1 -forms, so also on $m$-tuples of them. The invariance condition implies that the symmetry of the $m 1$-forms is preserved. This action persists at the limit when the $m$-tuple of 1 -forms is collapsed to the zero-section.

To compute the action, it is better to work in the idealic viewpoint. We imitate the steps from FT19 section 3.2.

Let $I$ be an ideal representing a $\mathfrak{g}$-complex structure. Write $I$ with generators $\left\langle f_{1}, \ldots, f_{r}\right\rangle$. Each $f_{k}$ can be considered as a function on $T^{* \mathbb{C}} \Sigma$, so its variation under a Hamiltonian $H$ is given by the Poisson bracket $\left\{H, f_{k}\right\}$. The tangent space at $I$ in the space of all ideals of codimension $m$ is the set of all ring homomorphisms from $I$ to $A / I$. Thus a Hamiltonian $H$ changes $I$ to $\left\langle f_{1}+\varepsilon\left\{H, f_{1}\right\} \bmod I, \ldots, f_{r}+\varepsilon\left\{H, f_{r}\right\}\right.$ $\bmod I\rangle$.

We restate a lemma from [FT19] (lemma 4) which allows to simplify $H$ :

Lemma 3.2. Let $I=\left\langle f_{1}, \ldots, f_{r}\right\rangle$ be an ideal of $\mathbb{C}[z, \bar{z}, p, \bar{p}]$ such that $\left\{f_{i}, f_{j}\right\}=0$ $\bmod I$ for all $i$ and $j$. Then for all polynomials $H$ and all $k \in\{1, \ldots, r\}$ we have $\left\{H, f_{k}\right\} \bmod I=\left\{H \bmod I, f_{k}\right\} \bmod I$.

Proof. The only thing to show is that if we replace $H$ by $H+g f_{l}$ for some polynomial $g$ and some $l \in\{1, \ldots, r\}$, the expression does not change. Indeed, $\left\{H+g f_{l}, f_{k}\right\}=$ $\left\{H, f_{k}\right\}+g\left\{f_{l}, f_{k}\right\}+\left\{g, f_{k}\right\} f_{l}=\left\{H, f_{k}\right\} \bmod I$ using the assumption.

Proposition 3.3. The ideals of $\operatorname{Hilb}_{0}^{\text {reg }}(\mathfrak{g})$ for $\mathfrak{g}$ classical satisfy the condition of the previous lemma.

Proof. For $A_{n}$, we have $I=\left\langle p^{n}, \bar{p}=\mu_{2} p+\ldots+\mu_{n} p^{n-1}=Q(p)\right\rangle$. We compute $\left\{p^{n},-\bar{p}+\right.$ $Q(p)\}=n p^{n-1} \partial Q=0 \bmod I$ since there is no constant term in $Q$.

The same argument holds for $B_{n}$ and $C_{n}$ since their ideals are special cases of the ideal of type $A_{n}$.

For $D_{n}$, the ideal $I$ is given by

$$
\begin{aligned}
\left\langle p^{2 n-1}, p \bar{p}\right. & =\mu_{2} p^{2}+\mu_{4} p^{4}+\ldots+\mu_{2 n-2} p^{2 n-2}=Q(p)+\mu_{2 n-2} p^{2 n-2}, \\
\bar{p}^{2} & \left.=\nu_{2} p^{2}+\nu_{4} p^{4}+\ldots+\nu_{2 n-2} p^{2 n-2}=R(p)+\nu_{2 n-2} p^{2 n-2}\right\rangle .
\end{aligned}
$$

As before the Poisson brackets with the first generator $p^{2 n-1}$ vanishes modulo $I$ since $Q$ and $R$ have no constant terms. To compute the last Poisson bracket, define $\tilde{Q}=Q / p$. By the relations in $I$, we have $R=\tilde{Q}^{2}+p^{2 n-2} \tilde{R}$ for some polynomial $\tilde{R}$ (see 
subsection 1.8). Remark further that $\left\{a(z, \bar{z}) p^{k} \bar{p}^{l}, b(z, \bar{z}) p^{k^{\prime}} \bar{p}^{l^{\prime}}\right\}=0 \bmod I$ whenever $k+l+k^{\prime}+l^{\prime}>n-1$ since any term of degree $n-1$ in $p$ and $\bar{p}$ is in $I$ and the Poisson bracket lowers this degree by 1 . With all this, we compute

$$
\begin{aligned}
& \left\{-p \bar{p}+Q+\mu_{2 n-2} p^{2 n-2},-\bar{p}^{2}+R+\nu_{2 n-2} p^{2 n-2}\right\} \\
= & \left\{-p \bar{p}+p \tilde{Q}+\mu_{2 n-2} p^{2 n-2},-\bar{p}^{2}+\tilde{Q}^{2}+p^{2 n-2}\left(\tilde{R}+\nu_{2 n-2}\right)\right\} \\
= & \left\{-p \bar{p}+p \tilde{Q},-\bar{p}^{2}+\tilde{Q}^{2}\right\} \\
= & 2 \bar{\partial} \tilde{Q}(p \bar{p}-p \tilde{Q})-2 \tilde{Q} \partial \tilde{Q}(\bar{p}-\tilde{Q}) \\
= & 2(p \bar{p}-Q)\left(\bar{\partial} \tilde{Q}-\frac{\tilde{Q}}{p} \partial \tilde{Q}\right) \\
= & 2 \mu_{2 n-2} p^{2 n-2}\left(\bar{\partial} \tilde{Q}-\frac{\tilde{Q}}{p} \partial \tilde{Q}\right) \\
= & 0
\end{aligned}
$$

where the last line comes from the fact that $p$ divides the polynomial $\bar{\partial} \tilde{Q}-\frac{\tilde{Q}}{p} \partial \tilde{Q}$.

As a consequence, when computing the action of a Hamiltonian $H$ on a $\mathfrak{g}$-complex structure, we can reduce it modulo $I$. In particular if $H \bmod I=0$, the higher diffeomorphism generated by $H$ does not act at all. For $\mathfrak{g}$ of type $A_{n}, B_{n}$ or $C_{n}$ we can reduce $H$ to a polynomial in $p$, and for $D_{n}$ we can reduce it to $H=w_{-} \bar{p}+$ $\sum_{k=0}^{n-2} w_{2 k+1} p^{2 k+1}$.

3.3. Local theory. Now, we can study the local theory of $\mathfrak{g}$-complex structures. Let $z_{0}$ be a point on $\Sigma$ and take a small chart around it which sends to the unit disk $\Delta$ in the complex plane (with $z_{0}$ send to the origin).

Theorem 3.4. For $\mathfrak{g}$ of type $A_{n}, B_{n}$ or $C_{n}$, the $\mathfrak{g}$-complex structure can be locally trivialized, i.e. there is a higher diffeomorphism of type $\mathfrak{g}$ which sends all higher Beltrami differentials to 0 for all small $z \in \mathbb{C}$.

For $\mathfrak{g}$ of type $D_{n}$, all $\mathfrak{g}$-complex structures with non-vanishing $\sigma_{n}$ on $\Delta$ are equivalent under higher diffeomorphisms. However, the zero locus of $\sigma_{n}$ on $\Delta$ is an invariant.

Proof. The proof for $\mathfrak{g}$ of type $A_{n}$ was done in [FT19] appendix 5.2, using a method in the spirit of the proof of Darboux's theorem in symplectic geometry.

If $\mathfrak{g}$ is of type $B_{n}$ or $C_{n}$, the standard representations realizes the $\mathfrak{g}$-complex structure as a substructure of type $A_{n}$. Since the last is trivializable, so is the $\mathfrak{g}$-complex structure in that case.

For $\mathfrak{g}$ of type $D_{n}$, we use the same method as for type $A_{n}$ by a Hamiltonian flow argument. We start with an ideal $I$ determined by higher Beltrami differentials $\left(\mu_{2}, \mu_{4}, \ldots, \mu_{2 n-2}, \nu_{2 n-2}\right)$. The action on $\mu_{2 i}$ is the same as for $\mathfrak{g}=\mathfrak{s l}_{2 n}$ so we can trivialize them using a Hamiltonian $H$ which is a polynomial in $p$. So we are left with

$$
I=\left\langle p^{2 n-1}, p \bar{p},-\bar{p}^{2}+\nu_{2 n-2} p^{2 n-2}\right\rangle .
$$

We have seen at the end of subsection 3.2 that in the case $D_{n}$, any Hamiltonian can be reduced to $H=w_{-} \bar{p}+\sum_{k=0}^{n-2} w_{2 k+1} p^{2 k+1}$. The only part of this Hamiltonian acting on $\nu_{2 n-2}$ is $H=w_{-} \bar{p}$, which also changes $\mu_{2 n-2}$. So in order to assure that $\mu_{2 n-2}$ stays zero, we use

$$
H=w_{-} \bar{p}+w_{2 n-3} p^{2 n-3} \text {. }
$$


We compute the action of this Hamiltonian on the ideal $I$. For the second generator of $I$ we get:

$$
\left\{w_{-} \bar{p}+w_{2 n-3} p^{2 n-3},-p \bar{p}\right\}=p^{2 n-2}\left(\bar{\partial} w_{2 n-3}+\partial w_{-} \nu_{2 n-2}\right) \quad \bmod I .
$$

For the third generator of $I$ we get

$$
\left\{w_{-} \bar{p}+w_{2 n-3} p^{2 n-3},-\bar{p}^{2}+\nu_{2 n-2} p^{2 n-2}\right\}=p^{2 n-2}\left(w_{-} \bar{\partial} \nu_{2 n-2}+2 \bar{\partial} w_{-} \nu_{2 n-2}\right) \quad \bmod I .
$$

Denote by $\mu_{2-2}^{t}$ and $\nu_{2 n-2}^{t}$ the image of $\mu_{2 n-2}$ and $\nu_{2 n-2}$ under the flow generated by $H$ at time $t$. From the above computation we get

$$
\left\{\begin{array}{l}
\frac{d}{d t} \mu_{2 n-2}^{t}=\bar{\partial} w_{2 n-3}+\partial w_{-} \nu_{2 n-2} \\
\frac{d}{d t} \nu_{2 n-2}^{t}=\left(w_{-} \bar{\partial}+2 \bar{\partial} w_{-}\right) \nu_{2 n-2}
\end{array}\right.
$$

Instead of keeping $\nu_{2 n-2}$, we work with the higher Beltrami differential $\sigma_{n}$. Since all the $\mu_{2 i}$ are zero in $I$, we have $\nu_{2 n-2}=\sigma_{n}^{2}$. Therefore we get from the second equation above $\frac{d}{d t}\left(\sigma_{n}^{2}\right)=\left(w_{-} \bar{\partial}+2 \bar{\partial} w_{-}\right)\left(\sigma_{n}^{2}\right)$ which gives

$$
\frac{d}{d t} \sigma_{n}^{t}=\bar{\partial}\left(w_{-}^{t} \sigma_{n}^{t}\right)
$$

We wish to have $\frac{d}{d t} \mu_{2 n-2}^{t}=0$ to stay with $\mu_{2 n-2}=0$. For $\sigma_{n}$, we show that we can deform it to the constant function 1 on the unit disk, assuming $\sigma_{n}$ vanishes nowhere on $\Delta$. We choose the path $\sigma_{n}^{t}=(1-t) \sigma_{n}^{0}+t$ from the initial $\sigma_{n}^{0}$ to the constant function 1 . If $\sigma_{n}^{t}=0$ for some $t$, we have to modify slightly the path. We get $\frac{d}{d t} \sigma_{n}^{t}=1-\sigma_{n}^{0}$.

Denote by $T$ the local inverse of the $\bar{\partial}$-operator, i.e. $\bar{\partial}(T f)=f=T \bar{\partial} f$ for all $f \in L^{2}(\Delta)$. The operator $T$ is a pseudo-differential operator given by

$$
T f(z)=\frac{1}{2 \pi i} \int_{\mathbb{C}} \frac{f(\zeta)}{\zeta-z} d \zeta \wedge d \bar{\zeta}
$$

We can solve equation (3.1) with $T$ :

$$
w_{-}^{t}=\frac{1}{\sigma_{n}^{t}} T\left(1-\sigma_{n}^{0}\right) .
$$

Putting this solution into the equation for $\frac{d}{d t} \mu_{2 n-2}^{t}$, we can solve for $w_{2 n-3}$ :

$$
w_{2 n-3}^{t}=-T\left(\partial w_{-}^{t} \nu_{2 n-2}^{t}\right) \text {. }
$$

Finally, we multiply $H$ by a bump function, a function on $\Delta$ which is 1 in a neighborhood of the origin and 0 outside a bigger neighborhood of the origin, which ensures that the Hamiltonian vector field is compactly supported, so it can be integrated to all times. In particular for $t=1$ we get $\sigma_{n}(z)=1$ for all $z$ near the origin.

The following argument shows that the zero locus of $\sigma_{n}$ can not be changed by a higher diffeomorphism: The ideal $\left\langle p^{2 n-1}, p \bar{p},-\bar{p}^{2}+\nu_{2 n-2} p^{2 n-2}\right\rangle$ is the deformation ideal of the singularity $-\frac{\nu_{2 n-2}}{2 n-1} p^{2 n-1}+p \bar{p}^{2}$ which is the Kleinian singularity of type $D_{2 n}$ if $\nu_{2 n-2} \neq 0$. In particular, this singularity is robust under diffeomorphisms, so is its deformation ideal.

Remark. It is interesting to notice the appearance of Kleinian singularities, which have an ADE-classification. The fact that for $\mathfrak{g}$ of type $D_{n}$ the singularity is of type $D_{2 n}$ is linked to the representation of $\mathfrak{s o}_{2 n}$ on $\mathbb{C}^{2 n}$. There should be a more intrinsic way to link $\mathfrak{g}$-complex structures to singularities of type $\mathfrak{g}$. 
An idea in this direction is the following: the singularity of type $\mathfrak{g}$ appears inside the Lie algebra $\mathfrak{g}$, more precisely inside the nilpotent variety along the subregular locus (see [St74]). A minimal resolution of this singularity is given by the Springer resolution. There should be a link between $\mathfrak{g}$-Hilbert schemes and the Springer resolution.

Since there are no local invariants for $\mathfrak{g}$-complex structures, only their global geometry is non-trivial.

3.4. Definition of the moduli space. To define the moduli space of $\mathfrak{g}$-complex structures, there is one more subtlety: in order to get one component, we have to fix an orientation on $\Sigma$. We then call a complex structure compatible if the induced orientation coincides with the given orientation on $\Sigma$. We call a $\mathfrak{g}$-complex structure compatible if the induced complex structure is.

Definition 3.5. The moduli space $\hat{\mathcal{T}}_{\mathfrak{g}} \Sigma$ is the space of all compatible $\mathfrak{g}$-complex structures modulo the action of higher diffeomorphisms of type $\mathfrak{g}$.

Notice that a $\mathfrak{g}$-complex structure is compatible iff $\mu_{2}(z) \bar{\mu}_{2}(z)<1$. Reverting the orientation on $\Sigma$ we get another copy of $\hat{\mathcal{T}}_{\mathfrak{g}} \Sigma$ corresponding to those $\mathfrak{g}$-complex structures with $\mu_{2}(z) \bar{\mu}_{2}(z)>1$.

For $\mathfrak{g}=\mathfrak{s l}_{2}$ we get Teichmüller space since we can reduce any Hamiltonian to $H=w(z, \bar{z}) p$ which generates a linear diffeomorphism of $T^{*} \Sigma$, coming from a diffeomorphism on $\Sigma$ isotopic to the identity.

The moduli space has the following properties:

Theorem 3.6. For $\mathfrak{g}$ of classical type, and a surface $\Sigma$ of genus $g \geq 2$ the moduli space $\hat{\mathcal{T}}_{\mathfrak{g}} \Sigma$ is a contractible manifold of complex dimension $(g-1) \operatorname{dim} \mathfrak{g}$. In addition, its cotangent space at any point I is given by

$$
T_{I}^{*} \hat{\mathcal{T}}_{\mathfrak{g}} \Sigma=\bigoplus_{m=1}^{r} H^{0}\left(\Sigma, K^{m_{i}+1}\right)
$$

where $\left(m_{1}, \ldots, m_{r}\right)$ are the exponents of $\mathfrak{g}$ and $r=\mathrm{rk} \mathfrak{g}$ denotes the rank of $\mathfrak{g}$.

Notice that the differentials in $H^{0}\left(\Sigma, K^{m_{i}+1}\right)$ are holomorphic with respect to the complex structure induced from the $\mathfrak{g}$-complex structure (see proposition 2.2).

Proof. The case for $A_{n}$ has been treated in [FT19], theorem 2. The cases $B_{n}$ and $C_{n}$ are exactly analogous:

One shows that at every point, the cotangent space exists and is of the form stated in the theorem. From this follows that $\hat{\mathcal{T}}_{\mathfrak{g}} \Sigma$ is a manifold. The only point to check is the appearance of the exponents of the Lie algebra. Since $\mu_{2 i}$ is a section of $K^{1-2 i} \otimes \bar{K}$ (see equation (2.1)) its dual $t_{2 i}$ is a section of $K^{2 i}$. Since the exponents for $B_{n}$ and $C_{n}$ are the same and equal to $(1,3, \ldots, 2 n-1)$, we get the desired form stated in the theorem.

For $\mathfrak{g}$ of type $D_{n}$, suppose first that the higher Beltrami differential $\sigma_{n}$ vanishes nowhere 1 . By the local theory, we know that there is a coordinate system in which $\mu_{2 i}=0$ for all $i=1, \ldots, n-1$. In that case, we know that the variation of $\mu_{2 i}$ under a higher diffeomorphism generated by $H=w_{-} \bar{p}+\sum_{k=0}^{n-2} w_{2 k+1} p^{2 k+1}$ is given by $\delta \mu_{2 i}=\bar{\partial} w_{2 i-1}$ and equation (3.1) gives $\delta \sigma_{n}=\bar{\partial}\left(w_{-} \sigma_{n}\right)$. The variation of $\mu_{2 i}$ is the

\footnotetext{
${ }^{1}$ This exists since $\sigma_{n}$ is a smooth section of $K^{1-n} \otimes \bar{K}$ which is of negative degree since $n>2$ and $g \geq 2$.
} 
same as in the case of type $A_{n}$, so we know that these contribute to the cotangent bundle by a term $H^{0}\left(\Sigma, K^{2 i}\right)$. For the term $\sigma_{n}$ we use the pairing between differential of type $(1-n, 1)$ and of type $(n, 0)$ given by integration over the surface. We get

$$
\begin{aligned}
\left(\left\{\delta \sigma_{n}\right\} / \bar{\partial}\left(w_{-} \sigma_{n}\right)\right)^{*} & =\left\{t_{n} \in \Gamma\left(K^{n}\right) \mid \int t_{n} \bar{\partial}\left(w_{-} \sigma_{n}\right)=0 \forall w_{-} \in \Gamma(\bar{K})\right\} \\
& =\left\{t_{n} \in \Gamma\left(K^{n}\right) \mid \int \bar{\partial} t_{n} w_{-} \sigma_{n}=0 \forall w_{-} \in \Gamma(\bar{K})\right\} \\
& =\left\{t_{n} \in \Gamma\left(K^{n}\right) \mid \bar{\partial} t_{n}=0\right\} \\
& =H^{0}\left(\Sigma, K^{n}\right)
\end{aligned}
$$

where we used that $\sigma_{n}$ vanishes nowhere.

Hence the cotangent bundle is given by

$$
T_{I}^{*} \hat{\mathcal{T}}_{\mathfrak{g}} \Sigma=\bigoplus_{m=1}^{n-1} H^{0}\left(\Sigma, K^{2 m}\right) \oplus H^{0}\left(\Sigma, K^{n}\right) .
$$

The exponents of $\mathfrak{s o}_{2 n}$ are precisely $(1,3, \ldots, 2 n-3, n-1)$, so the cotangent bundle is of the form stated in the theorem.

If $\sigma_{n}$ vanishes at some places, we can get $\sigma_{n}$ by a limit of non-vanishing $\sigma_{n}^{t}$ such that for $t \rightarrow 0$ we get $\sigma_{n}$. Since holomorphicity is a closed condition, we still have the same result.

For the dimension of $\hat{\mathcal{T}}_{\mathfrak{g}} \Sigma$, we use $\operatorname{dim} H^{0}\left(K^{m_{i}+1}\right)=(g-1)\left(2 m_{i}+1\right)$ by RiemannRoch (using $g \geq 2$ ). We get

$$
\operatorname{dim} \hat{\mathcal{T}}_{\mathfrak{g}} \Sigma=(g-1) \sum_{i=1}^{r}\left(2 m_{i}+1\right)=(g-1) \operatorname{dim} \mathfrak{g}
$$

using a well-known formula coming from the decomposition of $\mathfrak{g}$ as $\mathfrak{s l}_{2}$-module using the principal $\mathfrak{s l}_{2}$-triple.

Contractibility in all cases is easy: given an equivalence class of a $\mathfrak{g}$-complex structure by its set $S$ of higher Beltrami differentials, we can retract it in a direct linear way $t S$ to the structure where all Beltrami differentials are 0 . We use that if $S$ and $S^{\prime}$ are equivalent under a higher diffeomorphism, so are $t S$ and $t S^{\prime}$.

From the previous theorem, we see that our moduli space $\hat{\mathcal{T}}_{\mathfrak{g}} \Sigma$ shares a lot of properties with Hitchin's component, in particular the dimension and contractibility. There is another common property to notice:

Proposition 3.7. There is a map from Teichmüller space into the moduli space $\hat{\mathcal{T}}_{\mathfrak{g}} \Sigma$.

Proof. The proposition follows from the map $\psi: \operatorname{Hilb}\left(\mathfrak{s l}_{2}\right) \rightarrow \operatorname{Hilb}^{\text {reg }}(\mathfrak{g})$ constructed in equation (1.2) in section 1.4. This map restricts to a map between the zero-fibers and extends over the surface $\Sigma$. Finally the map descends to the quotient by higher diffeomorphisms since for $\mathfrak{s l}_{2}$ we only quotient by diffeomorphisms of $\Sigma$.

The same property holds for $G$-Hitchin components which can be defined as the deformation space of representations of the form $\pi_{1}(\Sigma) \rightarrow P S L_{2}(\mathbb{R}) \rightarrow G$ where the first map is a Fuchsian representation and the second one is the principal map. So inside the $G$-Hitchin component sits a copy of Teichmüller space. The same situation holds in our case, and the map $\psi$ is constructed using the principal map as well.

Of course, we conjecture the equivalence of Hitchin's component and the moduli space of $\mathfrak{g}$-complex structures: 
Conjecture 3.8. The moduli space $\hat{\mathcal{T}}_{\mathfrak{g}} \Sigma$ is canonically isomorphic to Hitchin's component in the character variety $\operatorname{Hom}\left(\pi_{1}(\Sigma), G\right) / G$ where $G$ is the real split Lie group associated to $\mathfrak{g}$.

3.5. Spectral curve. In this final part, we construct a spectral curve in $T^{\star \mathbb{C}} \Sigma$ associated to a cotangent vector to $\hat{\mathcal{T}}_{\mathfrak{g}}$, i.e. a $\mathfrak{g}$-complex structure and a set of holomorphic differentials. The case for $\mathfrak{g}$ of type $A_{n}$ was treated in [FT19], section 4.

In loc. cit., we proved that the zero-fiber $\operatorname{Hilb}_{0}^{n}\left(\mathbb{C}^{2}\right)$ is Lagrangian in the reduced Hilbert scheme $\mathrm{Hilb}_{\text {red }}^{n}\left(\mathbb{C}^{2}\right)$. This stays true for all classical $\mathfrak{g}$ :

Proposition 3.9. The regular zero-fiber $\operatorname{Hilb}_{0}^{\text {reg }}(\mathfrak{g})$ is a Lagrangian subspace of $\mathrm{Hilb}^{\text {reg }}(\mathfrak{g})$ for classical $\mathfrak{g}$.

Proof. Since we are in the regular part, proposition 1.8 gives a parametrization. For classical $\mathfrak{g}$, via the standard representation we can consider $\operatorname{Hilb}^{\text {reg }}(\mathfrak{g})$ as subset of $\mathrm{Hilb}_{\text {red }}^{m}\left(\mathbb{C}^{2}\right)$ which remains symplectic and we can explicitly check that the zero-fiber $\operatorname{Hilb}_{0}^{\text {reg }}(\mathfrak{g})$ is Lagrangian.

More generally, Haiman in his paper [Ha98] described a way to find coordinates of $\operatorname{Hilb}^{n}\left(\mathbb{C}^{2}\right)$ in the chart associated to a Young diagram $D$. For each box $B_{x} \in D$ consider the rightmost box $B_{r} \in D$ in the same row as $B_{x}$ and the bottommost box $B_{b} \in D$ in the same column as $B_{x}$ (see figure 4). The box $B_{r+1}$ to the right of $B_{r}$ is not in $D$, so gives a linear combination of boxes in $D$. Denote by $b_{x, r}$ the coefficient of $B_{b}$ in this linear combination. Similarly, denote by $b_{x, b}$ the coefficient of $B_{r}$ in the linear combination associated to the box $B_{b+1}$ at the bottom of $B_{b}$. Haiman shows that the set $\left\{b_{x, r}, b_{x, b}\right\}_{x \in D}$ is a coordinate system. We have even more:

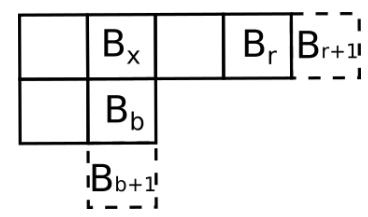

FiguRE 4. Haiman's coordinates

Proposition 3.10. Haiman's coordinates $\left\{b_{x, r}, b_{x, b}\right\}_{x \in D}$ are canonical coordinates with respect to the symplectic structure of the punctual Hilbert scheme.

Proof. The symplectic structure of the punctual Hilbert scheme comes from the canonical symplectic structure of $\mathbb{C}^{2 n}$ given by $\omega=\sum_{i} d x_{i} \wedge d y_{i}$. Consider the multiplication operators $M_{x}$ and $M_{y}$ in the quotient $\mathbb{C}[x, y] / I$ where $I$ is an element in the Hilbert scheme (idealic viewpoint). Diagonalizing these operators give diagonal matrices with entries $\left(x_{1}, \ldots, x_{n}\right)$ and $\left(y_{1}, \ldots, y_{n}\right)$. Changing to the base adapted to the Young diagram $D$ (basis generated by monomials $x^{i} y^{j}$ where $(i, j) \in D$ ), the matrix $M_{x}$ becomes a matrix $N_{x}$ with entries 1 on the line under the diagonal, apart from some columns where the linear combination associated to some $B_{r+1}$ is written. Similarly, the matrix $M_{y}$ becomes a matrix $N_{y}$ with entries 1 on the line under the diagonal, apart from some columns where the linear combination associated to some 
$B_{b+1}$ is written. Finally, we compute

$$
\begin{aligned}
\omega & =\operatorname{tr} d\left(\begin{array}{lll}
x_{1} & & \\
& \ddots & \\
& \ddots & x_{n}
\end{array}\right) \wedge d\left(\begin{array}{lll}
y_{1} & & \\
& \ddots & \\
& & y_{n}
\end{array}\right) \\
& =\operatorname{tr} d N_{x} \wedge d N_{y} \\
& =\sum_{x \in D} d b_{x, r} \wedge d b_{x, b} .
\end{aligned}
$$

As an application, we can use Haiman coordinates for the ideal with three generators for $\mathfrak{g}$ of type $D_{n}$ in subsection 1.8 . In particular, we see that the coordinates $u$ and $\nu_{2 n-2}$ are canonically conjugated.

For a modified smooth version of the $\mathfrak{g}$-Hilbert scheme, we conjecture the following:

Conjecture 3.11. The modified smooth version of the $\mathfrak{g}$-Hilbert scheme is symplectic and the zero-fiber is a Lagrangian subspace.

If we assume conjecture 1.16 true, stating that the modified version of the $\mathfrak{g}$ Hilbert scheme is a minimal resolution of $\mathfrak{h}^{2} / W$, we get a symplectic structure. Indeed $\mathfrak{h}^{2}=T^{*} \mathfrak{h}$ has a canonical symplectic structure, which is invariant under the action of $W$. Hence it lifts to the minimal resolution.

Now we construct the spectral curve. First, we look at $\mathfrak{g}$ of type $A_{n}, B_{n}$ or $C_{n}$. We can write a cotangent vector in $T^{\star} \hat{\mathcal{T}}_{\mathfrak{g}}$ as an equivalence class of higher Beltrami differentials $\mu_{i}$ and holomorphic differentials $t_{i}$. To write in a uniform way, set $\mu_{i}$ or $t_{i}$ to 0 whenever it does not appear for $\mathfrak{g}$. For example for type $B_{n}$ or $C_{n}$ all variables with odd index are 0 .

Associate polynomials $P(p)=p^{m}+\sum_{i} t_{i} p^{m-i}$ and $Q(p, \bar{p})=-\bar{p}+\sum_{i} \mu_{i} p^{i-1}$ (where $m$ is the dimension of the standard representation of $\mathfrak{g})$. Put $I=\langle P, Q\rangle$. Define the spectral curve $\tilde{\Sigma} \subset T^{*} \mathbb{C} \Sigma$ by the zero set of $P$ and $Q$. It is a ramified cover over $\Sigma$ with $m$ sheets.

For $\mathfrak{g}$ of type $D_{n}$, a generic point in the cotangent bundle $T^{*} \hat{\mathcal{T}}_{\mathfrak{g}}$ corresponds to the ideal

$$
I=\left\langle p^{2 n}+t_{2} p^{2 n-2}+\ldots+t_{2 n-2} p^{2}+\tau_{n}^{2},-\bar{p}+\mu_{2} p+\ldots+\mu_{2 n} p^{2 n-1}\right\rangle
$$

which can be seen as a special case of $A_{n}$. Thus we can proceed as above. In the case where $\tau_{n}=0$ we have seen in 1.8 that the ideal changes to an ideal with three generators. These generators still define a spectral curve in $T^{* \mathbb{C}} \Sigma$.

Proposition 3.12. The spectral curve $\tilde{\Sigma}$ is Lagrangian to order 1 in the holomorphic differentials $t$.

This is the precise analogue of proposition 5 in [FT19].

Proof. In the case where the ideal has two generators $P$ and $Q$ this is equivalent to $\{P, Q\}=0 \bmod I \bmod t^{2}$ for $I \in T^{*} \hat{\mathcal{T}}_{\mathfrak{g}}$. For $A_{n}$, the proof is given in loc. cit. For $B_{n}$ and $C_{n}$ it is completely analogous since the $\mathfrak{g}$-complex structure can be seen as a special case of $A_{n}$.

For $\mathfrak{g}$ of type $D_{n}$, a generic ideal has still two generators, so we have a special case of $A_{n}$. If the ideal has three generators, the spectral curve is still Lagrangian since it can be obtained as a limit of Lagrangian curves, and the property of being Lagrangian is closed. 
Since the spectral curve is Lagrangian to order 1 , the periods are well-defined up to this order. The ratios of these periods should give coordinates on $T^{\star} \hat{\mathcal{T}}_{\mathfrak{g}}$ and also on $\hat{\mathcal{T}}_{\mathfrak{g}}$. For the trivial $\mathfrak{g}$-complex structure (where all higher Beltrami differentials are 0$)$ we recover Hitchin's spectral curve.

Finally, we can recover the same spectral data as Hitchin in his paper on stable bundles [Hi87]. From a $\mathfrak{g}$-complex structure we get a bundle $V$ over the surface $\Sigma$ whose fiber at a point $z \in \Sigma$ is $\mathbb{C}[p, \bar{p}] / I(z)$ where we use the idealic viewpoint. We also get a line bundle $L$ on $\tilde{\Sigma}$ whose fiber is the eigenspace of $M_{p}$, the multiplication operator by $p$ in the quotient $\mathbb{C}[p, \bar{p}] / I$. This gives the spectral data for type $A_{n}$.

For $\mathfrak{g}$ of type $C_{n}$, we get in addition an involution $\sigma$ on the spectral curve $\tilde{\Sigma}$ given by $(p, \bar{p}) \mapsto(-p,-\bar{p})$. For $\mathfrak{g}$ of type $D_{n}$, the spectral curve is singular, having a double point. The spectral data is given by a desingularization of $\tilde{\Sigma}$, the involution $\sigma$ as for $C_{n}$ and the line bundle $L$. For $\mathfrak{g}$ of type $B_{n}$, there is a canonical subbundle $V_{0} \subset V=\mathbb{C}[p, \bar{p}] / I$ generated by the span of the image of $1 \in \mathbb{C}[p, \bar{p}]$ in the quotient $\mathbb{C}[p, \bar{p}] / I$ (since for $B_{n}$, we have $I \subset\langle p, \bar{p}\rangle$ ). Thus the vector bundle $V$ is an extension $V_{0} \rightarrow V \rightarrow V_{1}$. The spectral data is given by $\left(V_{0}, V_{1}, \sigma, L, \tilde{\Sigma}\right)$.

\section{Perspectives}

In addition to the various conjectures in this paper, we wish to give a conjectural larger picture which would imply the main conjecture 3.8 about the equivalence between Hitchin's component and our moduli space $\hat{\mathcal{T}}_{\mathfrak{g}}$.

Hitchin's original construction in [Hi92] of components in character varieties uses Higgs bundles and the hyperkähler structure of its moduli space $\mathcal{M}_{H}$. In one complex structure, say $I, \mathcal{M}_{H}$ has the complex structure from Higgs bundles. In all combinations of $J$ and $K$, it is the moduli space of flat $G^{\mathbb{C}}$-connections. The non-abelian Hodge correspondence is equivalent to the twistor description of this hyperkähler manifold. Hitchin constructs a fibration of $\mathcal{M}_{H}$ over a space of holomorphic differentials, whose fibers via the non-abelian Hodge correspondence give flat connections with monodromy in the split real group $G$.

There is a similar conjectural picture for $\mathfrak{g}$-complex structures: a hyperkähler manifold $\mathcal{M}$, which in complex structure $I$ is the cotangent space to the moduli space of $\mathfrak{g}$-complex structures $T^{*} \hat{\mathcal{T}}_{\mathfrak{g}} \Sigma$ and in all combinations of $J$ and $K$ is the moduli space of flat $G^{\mathbb{C}}$-connections. The analogue of Hitchin's fibration is simply the projection $\pi: T^{*} \hat{\mathcal{T}}_{\mathfrak{g}} \Sigma \rightarrow \hat{\mathcal{T}}_{\mathfrak{g}} \Sigma$. One has to prove an analogue of the non-abelian Hodge correspondence, i.e. a deformation of a pair ( $\mathfrak{g}$-complex structure, set of holomorphic differentials) to flat connections, and that the monodromy of the fibers of the projection $\pi$ lies in the split real group $G$.

The conception behind this analogy is the following: In Hitchin's case, we have a fixed complex structure on $\Sigma$ and a holomorphic Higgs field $\Phi \in H^{(1,0)}(\Sigma, \mathfrak{g})$ which gives a flat connection $\mathcal{A}(\lambda)=\lambda \Phi+A+\lambda^{-1} \Phi^{*}$. To get the Hitchin section, we choose a principal nilpotent element $f$ in the Lie algebra $\mathfrak{g}$ and deform it into the principal slice $f+Z(e)$. To avoid fixing a complex structure, we start with $\Phi=\Phi_{1} d z+\Phi_{2} d \bar{z}$. The flatness of $\mathcal{A}(\lambda)$ gives that $\Phi_{1}$ and $\Phi_{2}$ commute. We further impose $\Phi_{1}$ and $\Phi_{2}$ to be nilpotent. More specifically, we take $\Phi_{1}$ to be the principal nilpotent element $f$ and we choose $\Phi_{2} \in Z(f)$. Thus we have the same number of degrees of freedom 
as in the Higgs bundle setting. A pair of commuting nilpotent matrices of this form is precisely a point in $\operatorname{Hilb}_{0}^{r e g}(\mathfrak{g})$ which we used to construct $\mathfrak{g}$-complex structures.

\section{Appendix A. Punctual Hilbert schemes Revisited}

In this appendix, we review the punctual Hilbert scheme of the plane with its various viewpoints. Main references are Nakajima's book [Na99] and Haiman's paper Ha98.

A.1. Definition. To start, consider $n$ points in the plane $\mathbb{C}^{2}$ as an algebraic variety, i.e. defined by some ideal $I$ in $\mathbb{C}[x, y]$. Its function space $\mathbb{C}[x, y] / I$ is of dimension $n$, since a function on $n$ points is defined by its $n$ values. So the ideal $I$ is of codimension $n$. The space of all such ideals, or in more algebraic language, the space of all zero-subschemes of the plane of given length, is the punctual Hilbert scheme:

Definition A.1. The punctual Hilbert scheme $\operatorname{Hilb}^{n}\left(\mathbb{C}^{2}\right)$ of length $n$ of the plane is the set of ideals of $\mathbb{C}[x, y]$ of codimension $n$ :

$$
\operatorname{Hilb}^{n}\left(\mathbb{C}^{2}\right)=\{I \text { ideal of } \mathbb{C}[x, y] \mid \operatorname{dim}(\mathbb{C}[x, y] / I)=n\} \text {. }
$$

The subspace of $\mathrm{Hilb}^{n}\left(\mathbb{C}^{2}\right)$ consisting of all ideals supported at 0, i.e. whose associated algebraic variety is $(0,0)$, is called the zero-fiber of the punctual Hilbert scheme and is denoted by $\operatorname{Hilb}_{0}^{n}\left(\mathbb{C}^{2}\right)$.

A theorem of Grothendieck and Fogarty asserts that $\operatorname{Hilb}^{n}\left(\mathbb{C}^{2}\right)$ is a smooth and irreducible variety of dimension $2 n$ (see [Fo68]). The zero-fiber $\operatorname{Hilb}_{0}^{n}\left(\mathbb{C}^{2}\right)$ is an irreducible variety of dimension $n-1$, but it is in general not smooth.

A generic element of $\operatorname{Hilb}^{n}\left(\mathbb{C}^{2}\right)$, geometrically given by $n$ distinct points, is given by

$$
I=\left\langle x^{n}+t_{1} x^{n-1}+\cdots+t_{n},-y+\mu_{1}+\mu_{2} x+\ldots+\mu_{n} x^{n-1}\right\rangle .
$$

The second term can be seen as the Lagrange interpolation polynomial of the $n$ points.

A generic element of the zero-fiber is given by

$$
I=\left\langle x^{n},-y+\mu_{2} x+\ldots+\mu_{n} x^{n-1}\right\rangle .
$$

A.2. Resolution of singularities. Given an ideal $I$ of codimension $n$, we can associate its support, the algebraic variety defined by $I$, which is a collection of $n$ points (counted with multiplicity). The order of the points does not matter, so there is a map, called the Chow map, from $\operatorname{Hilb}^{n}\left(\mathbb{C}^{2}\right)$ to $\operatorname{Sym}^{n}\left(\mathbb{C}^{2}\right):=\left(\mathbb{C}^{2}\right)^{n} / \mathcal{S}_{n}$, the configuration space of $n$ points $\left(\mathcal{S}_{n}\right.$ denotes the symmetric group). A theorem of Fogarty asserts that the punctual Hilbert scheme is a minimal resolution of the configuration space.

In order to get a feeling for a general Lie algebra, notice that $n$ points of $\mathbb{C}^{2}$ is the same as two points in the Cartan $\mathfrak{h}$ of $\mathfrak{g l}_{n}$, and that the symmetric group is the Weyl group $W$ of $\mathfrak{g l}_{n}$. So the configuration space equals $\mathfrak{h}^{2} / W$ for $\mathfrak{g}=\mathfrak{g l}_{n}$. 
A.3. Matrix viewpoint. To an ideal $I$ of codimension $n$, we can associate two matrices: the multiplication operators $M_{x}$ and $M_{y}$, acting on the quotient $\mathbb{C}[x, y] / I$ by multiplication by $x$ and $y$ respectively. To be more precise, we can associate a conjugacy class of the pair: $\left[\left(M_{x}, M_{y}\right)\right]$.

The two matrices $M_{x}$ and $M_{y}$ commute and they admit a cyclic vector, the image of $1 \in \mathbb{C}[x, y]$ in the quotient (i.e. 1 under the action of both $M_{x}$ and $M_{y}$ generate the whole quotient).

Proposition A.2. There is a bijection between the Hilbert scheme and conjugacy classes of certain commuting matrices:

$$
\operatorname{Hilb}^{n}\left(\mathbb{C}^{2}\right) \cong\left\{(A, B) \in \mathfrak{g l}_{n}^{2} \mid[A, B]=0,(A, B) \text { admits a cyclic vector }\right\} / G L_{n}
$$

The inverse construction goes as follows: to a conjugacy class $[(A, B)]$, associate the ideal $I=\{P \in \mathbb{C}[x, y] \mid P(A, B)=0\}$, which is well-defined and of codimension $n$ (using the fact that $(A, B)$ admits a cyclic vector). For more details see [Na99].

It is this bijection which we use in the main text to generalize the punctual Hilbert scheme. Notice that the zero-fiber of the Hilbert scheme corresponds to nilpotent commuting matrices.

A.4. Reduced Hilbert scheme. We wish to define a subspace of $\operatorname{Hilb}^{n}\left(\mathbb{C}^{2}\right)$ corresponding to matrices in $\mathfrak{s l}_{n}$ in the matrix viewpoint. A generic point should be a pair of points in the Cartan $\mathfrak{h}$ of $\mathfrak{s l}_{n}$ modulo order. This corresponds to $n$ points in the plane with barycenter 0 .

Definition A.3. The reduced Hilbert scheme Hilb ${ }_{\text {red }}^{n}\left(\mathbb{C}^{2}\right)$ is the space of all elements of $\mathrm{Hilb}^{n}\left(\mathbb{C}^{2}\right)$ whose image under the Chow map ( $n$ points with multiplicity modulo order) has barycenter 0 .

With this definition, we get

\section{Proposition A.4.}

$$
\operatorname{Hilb}_{\text {red }}^{n}\left(\mathbb{C}^{2}\right) \cong\left\{(A, B) \in \mathfrak{s l}_{n}^{2} \mid[A, B]=0,(A, B) \text { admits a cyclic vector }\right\} / S L_{n} .
$$

Finally, it can be proven that the reduced Hilbert scheme is symplectic and that the zero-fiber $\operatorname{Hilb}_{0}^{n}\left(\mathbb{C}^{2}\right)$ is a Lagrangian subspace of Hilb red $\left(\mathbb{C}^{2}\right)$.

\section{Appendix B. Regular elements in SEMisimple Lie ALGEBRAS}

In this appendix, we gather all properties we need in the main text of regular elements in semisimple Lie algebras and we give precise references for these results. The main references are the books of Collingwood and McGovern [CM93], Steinberg [St74] and Humphreys [Hu95], as well as the papers [Ko59] and [Ko63] by Kostant.

Definition B.1. An element $x \in \mathfrak{g}$ is called regular if the dimension of its centralizer $Z(x)$ is equal to the rank of the Lie algebra $\mathrm{rk}(\mathfrak{g})$. A regular nilpotent element is called principal nilpotent.

Remark. Notice that in older literature, regular elements are defined in another way, using the characteristic polynomial of the adjoint map. The "old" notion includes only semisimple regular (in the sens above) elements.

The condition that the dimension of the centralizer has to be equal to the rank, does not come from nowhere: in fact it is the minimal possible dimension. 
Proposition B.2. For any $x \in \mathfrak{g}$, we have $\operatorname{dim} Z(x) \geq \operatorname{rk}(\mathfrak{g})$.

See for example lemma 2.1.15. in [CM93].

For the Lie algebras $\mathfrak{g l}_{n}$ and $\mathfrak{s l}_{n}$, we have the following characterization of regular elements from Steinberg [St74], proposition 2 in section 3.5:

Proposition B.3. For $\mathfrak{g}=\mathfrak{g l}_{n}$ or $\mathfrak{s l}_{n}$ and $x \in \mathfrak{g}$, we have the following equivalence: $x$ is regular $\Leftrightarrow \mu_{x}=\chi_{x} \Leftrightarrow x$ admits a cyclic vector

where $\mu_{x}$ and $\chi_{x}$ denote respectively the minimal and the characteristic polynomial of $x$, seen as a matrix.

Let us turn to the study of regular elements which are nilpotent.

Theorem B.4. There is a unique open dense orbit in the nilpotent variety consisting of principal nilpotent elements.

The original proof is due to Kostant, see corollary 5.5. in [Ko59]. See also theorem 4.1.6. in CM93.

There is a useful characterization of principal nilpotent elements in coordinates. For this, fix a root system $R$, fix a direction giving positive roots $R_{+}$. Denote by $\mathfrak{n}$ the positive nilpotent elements (upper triangular for $\mathfrak{s l}_{n}$ ).

Proposition B.5. Let $A \in \mathfrak{n}$. Then $A$ is principal nilpotent iff $\alpha(A) \neq 0$ for all simple roots $\alpha$.

The group version of this can be found in section 3.7. of [St74].

For a principal nilpotent element $f$, its centralizer $Z(f)$ has properties quite analogous to a Cartan, the centralizer of a regular semisimple element:

Theorem B.6. For $f$ a principal nilpotent element, its centralizer $Z(f)$ is abelian and nilpotent.

Kostant proves even more, using a limit argument: for any element $x \in \mathfrak{g}$, there is an abelian subalgebra of $Z(x)$ of dimension $\mathrm{rk} \mathfrak{g}$, see [Ko59], theorem 5.7. The nilpotency of $Z(f)$ can be found in [St74], corollary in section 3.7. The more precise structure of $Z(x)$ for any nilpotent $x$ is described in [CM93, section 3.4.

A principal nilpotent element permits to give a preferred representative of a conjugacy class of regular elements. Given $f$ principal nilpotent, denote by $e$ the other nilpotent element in a principal $\mathfrak{s l}_{2}$-triple constructed from $f$ (see Kostant [Ko59]). Then we get

Proposition B.7. Any regular orbit intersects $f+Z(e)$ in a unique point. So we have $\mathfrak{g}^{\text {reg }} / G \cong f+Z(e)$.

This follows from Lemma 10 of [Ko63]. The set $f+Z(e)$ is called a principal slice of $\mathfrak{g}$ (also Kostant section).

We are now going to "double" the previous setting. Define the commuting variety to be $\operatorname{Comm}(\mathfrak{g}):=\left\{(A, B) \in \mathfrak{g}^{2} \mid[A, B]=0\right\}$.

Theorem B.8 (Richardson). The set of commuting semisimple elements is dense in the commuting variety $\operatorname{Comm}(\mathfrak{g})$.

See the paper of Richardson [Ri79] for a proof. As a consequence, $\operatorname{Comm}(\mathfrak{g})$ is an irreducible variety, but highly singular.

With this, we can explore the minimal dimension of a centralizer of a commuting pair: 
Proposition B.9. For $(A, B) \in \operatorname{Comm}(\mathfrak{g})$, we have $\operatorname{dim} Z(A, B) \geq \mathrm{rk} \mathfrak{g}$.

Proof. Consider the set $M$ of elements with centralizer of minimal dimension. Since

$$
M=\left\{(A, B) \in \operatorname{Comm}(\mathfrak{g}) \mid \operatorname{rk}\left(a d_{A}, a d_{B}\right) \text { maximal }\right\}
$$

we see that $M$ is Zariski-open. By the theorem of Richardson it intersects the space of semisimple pairs for which the common centralizer is a Cartan $\mathfrak{h}$, so of dimension rk $\mathfrak{g}$.

\section{REFERENCES}

[CM93] David H. Collingwood and William M. McGovern: Nilpotent Orbits in Semisimple Lie Algebras, Van Nostrand Reinhold Math. Series, New York, 1993

[FT19] Vladimir V. Fock and Alexander Thomas: Higher complex structures, accepted at IMRN journal, https://arxiv.org/abs/1812.11199

[Fo68] John Fogarty: Algebraic families on an algebraic surface, Amer. J. Math. Vol. 90 No. 2 (1968), p. $511-521$

[Gi99] Victor Ginzburg: Principal Nilpotent pairs in a semisimple Lie algebras I, https://arxiv.org/abs/math/9903059

[Ha98] Mark Haiman: t,q-Catal numbers and the Hilbert scheme, Discrete Mathematics 193 (1998), pp. 201-224

[Hi92] Nigel Hitchin: Lie Groups and Teichmüller Space, Topology Vol. 31, No.3 (1992), p. 449 473

[Hi87] Nigel Hitchin: Stable Bundles and Integrable Systems, Duke Math. J. Vol. 54 No. 1 (1987), p. 91-114

[Hu95] James E. Humphreys: Conjugacy Classes in Semisimple Algebraic Groups, Math. Surveys and Monographs Vol. 43 (1995), AMS

[Ia72] Anthony Iarrobino: Punctual Hilbert schemes, Bull. AMS Vol. 78, No. 5 (1972), p. 819 823

[Ko59] Bertram Kostant: The principal three-dimensional subgroup and the Betti numbers of a complex simple Lie group, Amer. J. Math. 81 (1959), p. 973-1032

[Ko63] Bertram Kostant: Lie group representations on polynomial rings, Amer. J. Math. 85 (1963), p. $327-404$

[Na99] Hiraku Nakajima: Lectures on Hilbert Schemes of Points on Surfaces, University Lecture Series 18, AMS, 1999

[Ri79] R. William Richardson:Commuting varieties of semisimple Lie algebras and algebraic groups, Comp. Math., Tome 38 (1979) no. 3, p. 311-327

[St74] Robert Steinberg: Conjugacy Classes in Algebraic Groups, Lecture Notes in Mathematics 366, Springer-Verlag, New York, 1974 